\title{
Micromechanical properties of strain-sensitive lyriform organs of a wandering spider (Cupiennius salei).
}

Seth L. Young, ${ }^{a}$ Marius Chyasnavichyus, ${ }^{a}$ Friedrich G. Barth, ${ }^{b}, c$ Igor Zlotnikov, ${ }^{c}$ Yael Politi,c and Vladimir V. Tsukruka,*

a School of Materials Science and Engineering, Georgia Institute of Technology, Atlanta, Georgia 30332, United States of America

${ }^{b}$ Department of Neurobiology, University of Vienna, 1090 Vienna, Austria.

${ }^{c}$ Department of Biomaterials, Max Planck Institute of Colloids and Interfaces, Research Campus Golm, 14424 Potsdam, Germany.

\begin{abstract}
Highly sensitive lyriform organs located on the legs of the wandering spider Cupiennius salei allow the spider to detect nanometer-scale strains in the exoskeleton resulting from locomotion or substrate vibrations. Morphological features of the lyriform organs result in their specialization and selective sensitivity to specific mechanical stimuli, which make them interesting for bioinspired strain sensors. Here we utilize atomic force microscopy (AFM)-based force spectroscopy to probe nano-scale mechanical properties of the covering membrane of two lyriform organs found on Cupiennius salei: the vibration sensitive metatarsal lyriform organ (HS10) and the proprioreceptive tibial lyriform organ (HS8). Force distance curves (FDCs) obtained from AFM measurements displayed characteristic multi-layer structure behavior, with calculated elastic moduli ranging from $150 \mathrm{MPa}$ to $500 \mathrm{MPa}$ for different regions and indentation depths. In addition, we probed the lyriform organs with a large radius tip, which allowed for probing structural deformation by the application of high forces and large scale deformations without damaging the surface. The viscoelastic behavior of the sensor materials observed in this probing suggests mechanical relaxation times potentially playing a role in the time-dependent behavior of the lyriform organs.
\end{abstract}

Keywords: Mechanosensing, Epicuticle mechanics, Relaxation time, Force spectroscopy, Biosensing, Cupiennius salei

${ }^{*}$ Corresponding author: Tel.: 404-894-6081; Fax: 404-385-3112; Email address: vladimir@mse.gatech.edu 


\section{Introduction}

In the life of the wandering spider Cupiennius salei perception is dominated by mechanosensory systems [1]. These systems include tactile hairs [2,3,4], air-flow sensing trichobothria $[5,6,7,8]$, and slit sensilla embedded in the exoskeleton (Fig. 1) $[9,10,11,12]$. Of the $~ 3300$ slit sensilla known, about half form 144 closely packed groups of parallel slits known as lyriform organs (a name derived from the ancient musical instrument which they resemble, examples shown in Fig. 1B,C). Most of the lyriform organs are located near joints on the legs of the spider [13]. An interesting facet of the lyriform organs is their specialization in regard to their tuning to specific mechanical stimuli. Depending on several morphological factors including slit arrangement within an organ (e.g. angles between slits, length gradients, and lateral shifts amongst the slits), orientation of the organ with respect to the leg axis, dendrite position within the slits, and surrounding auxiliary structures (e.g. the cuticular pad in front of HS10; $[17,26]$ ), a single organ can be "tuned" to respond well to different stimulus parameters like strain direction or a particularly wide range of strain amplitudes, and also be sensitive to specific frequency ranges $[9,14,15,16,17,18]$. Because of this behavior, these slit sensory systems as well as their insect analogues, the campaniform sensilla, have become particularly interesting as models of biomimetic and bioinspired mechanosensors. There have been recently proposed uses of such bioinspired devices serving as embeddable, tunable strain sensors for space exploration applications and for easily created strain sensing in flexible electronics $[19,20,21,22,23,24]$. However, to better understand how these organs can be applied to solve prospective problems, a fundamental understanding of their materials properties is still required.

Recently, we have studied in detail the time- and temperature-dependent micromechanical properties of the epicuticular layer covering the metatarsal pad which serves to enhance the response of the vibration sensitive lyriform organ HS10 to high frequency stimuli $[17,25]$. Additionally, we have analyzed a variety of chitin fibril orientations and sclerotization levels resulting in a wide range of mechanical properties in the subsurface structures of the pad [26]. The pad was also found to play a key role 
in HS10 compression (stimulation) including the transformation of large initial deformations caused by the tarsus (tens of $\mu \mathrm{m}$ ) to much smaller deformations (tens of $\mathrm{nm})$ [26]. Notwithstanding the growing knowledge on the biomechanical properties of cuticular auxiliary structures such as the metatarsal pad, insight into crack deformational behavior provided by modeling experiments $[9,15,16,18]$, and knowledge of the sensory cells' response based on direct electrophysiological investigations [14,27], there is very little direct micromechanical probing of the lyriform organs themselves.

Each individual slit is an anisotropic hole in the exocuticle 1-2 $\mu \mathrm{m}$ wide and from $\sim 10$ $200 \mu \mathrm{m}$ long (cross-sectional schematic presented in Fig. 2). An epicuticular outer membrane covers the slit. Within the slit there is a viscous sodium-rich receptor lymph [28]. An inner membrane separates the bottom of the slit from the receptor lymph space below the slit (in the hypodermis) which is penetrated by the two dendrites of the sensory cells on their way to the slit. Whereas one of the dendrites attaches to the slit's outer membrane the other one ends just below the inner membrane. To date the particular role and functional meaning of the second shorter dendrite and the mechanical implications imposed by the inner membrane are not fully understood yet [27]. As forces compress the slit, the outer membrane deflects downward deforming the dendrite, which in turn results in the firing of action potentials. In this way, each slit is able to monitor strain in the exoskeleton of the spider through compressions of the slit on the order of $10^{-9} \mathrm{~m}$, caused by locomotion, changes in haemolymph pressure, and substrate vibrations caused by nearby prey and mates (see [18]). Obviously the geometrical and micromechanical properties of the outer membrane are critical in the functional performance and sensitivity of the lyriform organs, and as such merit careful investigation of their micromechanical properties, going beyond previous modeling efforts $[29,30]$.

Herein we present results of a micromechanical characterization of the surface of two lyriform organs of $C$. salei: (i) the metatarsal lyriform organ, HS10, because of its unique sensitivity to vibrational stimuli, and (ii) a proprioreceptive tibial lyriform organ, HS8, because of the large width and shallow depth of several of its slits which allow for easy access to measure the properties of the outer membrane. Additionally, a significant 
amount of literature on the HS8 organ facilitates the comparison between the mechanical properties observed here and the electrophysiological responses recorded earlier [1]. We utilize atomic force microscopy (AFM) and related surface force spectroscopy (SFS) to study geometrical and micromechanical properties of these organs with precise lateral control as well as in a controlled environment on living and rehydrated cuticle samples. Force distance curves (FDCs) were taken using both asreceived sharp AFM tips (radius of curvature around $10 \mathrm{~nm}$ ) as well as modified AFM tips with large radii of curvature (around 200-500 nm). These tips were used to investigate the mechanical properties of the surface of the epicuticular outer membrane (small radius tips) as well as the membrane structural modulus (large radius tips) at different penetration depths. This analysis confirmed a multi-layered structure with a softer viscoelastic layer on top of a stiff supporting layer, with elastic modulus values ranging from approximately $150 \mathrm{MPa}$ at the smallest penetration depths, to as high as 2 GPa for the highest applied forces obtained using a blunt AFM tip. Finally, we discuss how the viscoelastic nature of the upper layers could potentially contribute to the physiological response characteristics of the spider's mechanosensitive organs.

\section{Materials and Methods}

2.1 Materials. For measurements of the HS10 lyriform organ, live adult female wandering spiders (C. salei, Ctenidae) were received from the breeding stock of the Department of Neurobiology, University of Vienna. The spiders were anesthetized using $\mathrm{CO}_{2}$ gas and then attached to a removable AFM chuck by point-gluing the femoral, tibial, and metatarsal leg segments, with a 1:1 weight mixture of beeswax (Sigma Aldrich) and rosin (colophony, Alfa Aesar), carefully avoiding contact with or covering the lyriform organs of interest. In order to expose the organs for investigation with AFM, a majority of the hairs surrounding the lyriform organs were removed by gently rubbing a Kimwipe against the grain of the hairs, while those closest to the organ were carefully plucked using fine-tipped tweezers under a stereomicroscope avoiding damage and preserving the natural state of the cuticle. 
Measurements were performed on living intact spiders, freshly autotomized legs, as well as on stored material in some cases (as discussed below). Specifically, because of the location on the posterior side of the leg, FDCs on the HS8 lyriform organ could not be taken while the leg was still attached to the spider. Therefore freshly autotomized legs were obtained by slightly squeezing the femur with forceps as close to the cephalothorax as possible. Legs were also dissected from a spider which had been stored in $70 \% \mathrm{EtOH}$ solution and used for measurement. Briefly, the legs were removed at the femur-patella joint and washed thoroughly with ultra-pure water (18.2 $M \Omega \cdot c m$, Synergy UV-R, Millipore).

The opening of the leg was sealed at its proximal end with a droplet of beeswax/rosin mixture and the legs were stored in a refrigerator $\left(2-5^{\circ} \mathrm{C}\right)$ in a $4 \mathrm{ml}$ glass vial partially filled with spider saline solution. Spider saline solution was made according to the recipe outlined by Maier et al. [31] and Höger et al. [32]. $223 \mathrm{mM} \mathrm{NaCl}, 6.8 \mathrm{mM} \mathrm{KCl}, 8.0$ $\mathrm{mM} \mathrm{CaCl}_{2}$, and $5.1 \mathrm{mM} \mathrm{MgCl} 2$ were dissolved in Tris-HCl buffer, $\mathrm{pH}$ 7.8. The dissected legs were inspected visually for signs of biofouling daily, with no visual or AFM evidence of biofouling being observed throughout the experiments. In order to ensure that the cuticle of these dissected legs was as close to its natural state as possible, prior to measurement spider saline solution was gently injected into the spider leg at the site of the dissection (with just enough pressure to slightly extend the metatarsal joint). The leg was placed back into the glass vial partially filled with saline solution and allowed to equilibrate to room temperature. Immediately before scanning the surface of the spider leg was rinsed with ultrapure water to protect the AFM scanner from the corrosive saline solution.

2.2 Optical microscopy. Optical images were taken using a BX-51 microscope with a Dage-MTI XLM high-resolution cooled digital camera at 50x magnification under darkfield illumination. As described previously [25], due to the irregular shape of our samples and the inherent lack of depth of field associated with optical microscopy, each optical image presented here was digitally enhanced using focus stacking. Many images (>25) were recorded at different focal planes and the in-focus portions of each image superimposed using Helicon Focus version 5.3.14. 
2.3 Atomic force microscopy. All AFM measurements were made using a Bruker Icon AFM (Bruker) equipped with a Nanoscope $V$ controller. Rectangular n-type Si cantilevers with nominal spring constants of $0.2 \mathrm{~N} \mathrm{~m}^{-1}, 7.0 \mathrm{~N} \mathrm{~m}^{-1}$, and $80 \mathrm{~N} \mathrm{~m}^{-1}$ were obtained from MikroMasch (Lady's Island, SC, USA). For some experiments, we utilized probes with moderately-sized tip radii ( 200-500 $\mathrm{nm}$ radius), created by annealing as-received AFM tips at $1100{ }^{\circ} \mathrm{C}$ in air for $6-12$ hours. The radius of curvature of each tip was determined by scanning a titanium roughness sample (Bruker) and utilizing the blind tip estimation algorithm to create a height image of the reconstructed tip shape. This height image was sectioned and analyzed numerically, as described below. In the case of annealed AFM tips, the blind tip estimation method was used in combination with SEM in order to determine tip shape. Figure 3 shows topographical scans of the standard rough titania sample with clear differences resulting from tip convolution after annealing.

Cantilever deflection sensitivity was determined by averaging cantilever deflection versus z-piezo displacement curves taken on a clean sapphire crystal. The spring constant of each cantilever was determined by the thermal tuning method [33,34]. All topographical imaging was taken by using PeakForce Quantitative NanoMechanics (PFQNM) module, which utilizes a pulsed-force tapping mode. Briefly, the cantilever was oscillated at $2 \mathrm{kHz}$ and pressed into the sample surface during each down stroke, using a user-defined set-point of the applied force $(10-20 \mathrm{nN})$ as feedback control. The $x-y$ closed loop system incorporated on the Bruker Icon allows for precise piezo offset in the $x-y$ plane with insignificant piezo drift. Therefore in nearly all cases, mechanical properties were assessed via FDCs taken by zooming into a region and using the ramp mode within the PF-QNM module.

2.4 Electron microscopy. Scanning electron microscopy (SEM) images were collected on a Hitachi S-3400N thermal emission SEM. AFM tips were placed in a spring loaded clamp and scanned under vacuum at magnifications of $\sim 25 \mathrm{k}$ and $\sim 100 \mathrm{k}$ with an accelerating voltage of $5 \mathrm{kV}$. Further modification of SEM images of AFM tips used in Fig. 10 consisted of background removal using Adobe Photoshop. In these cases some minor fine resolution features were lost near the very tip of the probe, but overall shape 
and size was preserved to give the reader an accurate feel for the size of the tip-surface interactions occurring in these studies.

\section{Calculations}

3.1 Determination of tip radius. The radius of curvature, $R$, of a simple polynomial curve can be determined by Eq. 1:

$$
y=y(x) \rightarrow R=\frac{\left(1+\dot{y}^{2}\right)^{3 / 2}}{|\ddot{y}|}
$$

where $\dot{y}$ and $\ddot{y}$ represent the first- and second-order differentiations of $y(x)$ with respect to $x$, respectively. For simplicity here we assume an axisymmetric tip shape with a parabolic cross section described by the equation $y=A+B x+C x^{2}$. Section profile data from reconstructed tip images were evaluated using a MatLab-based, in-house developed data analysis program (MicroMechanical Analysis of Soft Polymeric Materials, MMA-SPM) and fit with a parabola under non-linear optimization conditions, in order to determine the second order polynomial coefficients. These coefficients were then used to determine tip radius, according to Eqn. 2:

$$
R_{\text {tip }}=\frac{\left(1+(B+2 C x)^{2}\right)^{3 / 2}}{|2 C|}=\frac{1}{2 C}
$$

It should be noted here that $x=\frac{-B}{2 C}$ at the apex of the tip, which results in a tip radius of $\frac{1}{2 C}$. Prior to each measurement, a reference Ti roughness sample (obtained from Bruker) was scanned and a blind-tip estimation algorithm was used in order to reconstruct a 3-dimesional estimation of tip shape. Several sectional profiles from this reconstruction were taken and this data was fitted according to Eqn. 1 and Eqn. 2 in order to determine the radius of curvature for each tip apex (e.g. tip apex profile fit in Fig. 4). In the case of annealed tips, we also confirmed our estimation via SEM imaging (see Fig. 3). 
3.2 Determination of elastic modulus. The elastic modulus of the sample surface was determined by analyzing FDCs which had low background noise and a clear contact point. Generally, more than 30 FDCs were collected at different locations and the approach portion of each FDC was used to determine the elastic modulus by fitting with the classical Sneddon equation for the purely elastic deformation of an elastic half space with a rigid paraboloidal punch $[35,36]$ :

$$
P=\left(\frac{4}{3} \frac{E}{1-v^{2}} R_{e q v}{ }^{1 / 2}\right) \delta^{3 / 2}
$$

where $P$ is the applied force, $R_{\text {eqv }}$ is the radius of curvature, $\delta$ is the penetration depth, $v$ is Poisson's ratio (chosen to be $0.3[7,25]$ ), and $E$ is the elastic modulus of the outermost surface layers.

3.3 Effect of surface topography. In the vast majority of AFM-based micromechanical measurements found in the literature, a sharp AFM probe is pushed into surfaces which are flat with low surface roughness. In this case, the equivalent contact radius is taken to be the radius of curvature of the tip (i.e. sphere on sphere of infinite radius). However, in the present experiments, the surface of the spider cuticle is rough with many ridges and valleys (see Fig. 4A). Therefore, we consider the curvature of the surface to determine whether the equivalent contact radius has a significant effect on the calculated elastic modulus. Figure $4 \mathrm{~B}$ shows section profiles taken along the dotted lines in the high-resolution height image presented in Fig. 4A. From these we determined that we can treat our contact problem as a rigid parabolic punch on a cylindrical surface, resulting in an elliptical contact area with major and minor axes, A and $B$, respectively. As a result, the equivalent contact radius can be calculated by Eqn. 4:

$$
R_{\text {eqv }}=\frac{1}{2}(A B)^{1 / 2}=\frac{1}{2}\left\{\frac{1}{4}\left(\frac{1}{\left(R_{1}^{\prime}\right)^{2}}+\frac{1}{R_{1}^{\prime} R_{2}^{\prime \prime}}\right)\right\}^{-1 / 2}
$$

where $R_{1}{ }^{\prime}$ is the major semi-axis of the indenter, and $R_{2}{ }^{\prime \prime}$ is the minor semi-axis of the surface. Inclusion of the equivalent radius slightly changes the relationship between applied force and penetration depth to: 


$$
\delta=\left(\frac{9}{16} \frac{P^{2}}{R_{e q v} E^{* 2}}\right)^{1 / 3} F_{2}\left(\frac{R^{\prime}}{R^{\prime \prime}}\right)
$$

where $R^{\prime}$ is the equivalent radius of the indenter, $R^{\prime \prime}$ is the equivalent radius of the

surface, and $F_{2}\left(\frac{R^{\prime}}{R^{\prime \prime}}\right)$ is a tabulated function which can be found in various literature [36] and more convenient electronic sources [37]. In the case presented in Fig. 4 we found that taking into account the curvature of the ridges resulted in a calculated elastic modulus of $162 \pm 34 \mathrm{MPa}$, while considering the surface a flat, semi-infinite half-space resulted in a calculated elastic modulus of $155 \pm 27 \mathrm{MPa}$. Because these values are in good agreement and within one standard deviation, we propose that with careful measurement (i.e. taking FDCs at the peak of ridges, and ensuring the entire sample is not significantly tilted) the elastic modulus of the surface of the spider legs can be reasonably well determined without additional data treatment.

3.4 Statistical Analysis. In the course of these studies total of three spiders were used two which were live (freshly autotomized legs were also used from these two) and one which was stored in $70 \%$ EtOH solution, as described above. For each of the spiders 45 legs were used to gather data from both the HS10 and HS8 lyriform organs. These data were pooled together to compute the elastic modulus of the spider lyriform organs described in the text. Data is presented in the text as the average \pm one standard deviation.

\section{Results}

4.1 AFM topographical imaging of lyriform organs. Figure 5 shows AFM topographical images of the metatarsal lyriform organ HS10 taken on a live female spider. Both height images show a wavy morphology that occurs naturally as the epicuticle is formed $[38,39]$. The cross-sectional profiles taken along the organ surface as indicated by the dashed lines superposed on the topographical data are presented in Figs. 5B and 5D. In each of these profiles the slits end in sharp points, which are a result of tip shape rather than membrane topography. Therefore the slit sensilla in HS10 are too narrow 
and deep for the AFM tip to reliably reach the outer membrane for mechanical measurements. However, because this is the organ of interest for us, we found it informative to measure the cuticle surrounding the slit outer membrane (i.e. the areas in-between slits, referred to as "surrounding cuticle" or "SC" below, seen in Figs. 5A,B) and to use the results for a comparison with organs with an outer membrane accessible to AFM. It should also be emphasized here that measurements on HS10 were made on a live spider. Thus in addition to an organ to organ comparison, these measurements served as a control for comparisons with rehydrated HS8 organs.

Figure 6 shows topographical AFM data of the tibial lyriform organ HS8, taken from a freshly autotomized leg. In these images, slits 8 and 7 (see Fig.1B) are present, and it can be seen there is also a similar wavy morphology, with wider ridges occurring outside of the organ, thinner ridges appearing on the cuticle in between each slit, and low roughness (i.e. no ridges) on the surface of the outer membrane spanning the slit (see Fig. 2). Also observable in Fig. 6 are the tips of the dendrites responsible for firing action potentials during compression of the slit under biologically-relevant loading.

4.2 Micromechanical properties of lyriform organs. Careful FDC measurements were taken on slit 8 in the HS8 organ of a freshly autotomized leg using an as-received AFM tip (radius of curvature $\sim 10-20 \mathrm{~nm}$ ). Representative curves are presented in Fig. 7 . It should be noted here that these measurements were taken using a tip with a very low spring constant, which results in the probing of only the first few $\mathrm{nm}$ depth from the surface. Greater probing depths yield different results (discussed below). In general the slope of the FDC is indicative of the elastic modulus of the material surface which is being measured.

In Fig. 7B it can be seen that while slopes of the FDCs taken at the surface of the cuticle in between the slits and the outer membrane surface are similar, the slope of the FDC taken at the site of the dendrite is noticeably different. The elastic modulus of the surrounding cuticle is slightly larger than the calculated elastic modulus of the surface of the outer membrane. However, both these elastic moduli are significantly larger than the elastic modulus calculated for the dendrite tip exposed at the surface. We also took 
FDCs on the accessible surface of the cuticle surrounding HS10. Due to the angle of the HS10 organ with respect to the long axis of the leg (see section image in ref. [7]) attention was paid to the slope at which the AFM was scanning to ensure that measurements were taken on surfaces which have low slope (determined by unprocessed AFM data). FDC collection and analysis assumes that the applied force is normal to the sample surface. Therefore scanning on high slope (highly angled with respect to the $x$-y plane) produced significant artifacts in the FDC data. The calculated elastic modulus of the surface of the cuticle surrounding HS10 was not statistically different from the values calculated from the HS8 organ. Therefore we suggest that the materials properties addressed in the present study are equivalent for different lyriform organs. Table 1 summarizes the values found.

Table 1: Summary of elastic moduli calculated from force distance curves obtained on HS8 and HS10 lyriform organs of $C$. Salei. In each case more than 30 curves were taken and the resulting calculation is presented as average \pm one standard deviation.

\begin{tabular}{|c|c|c|c|}
\hline $\begin{array}{c}\text { HS8 - Surrounding } \\
\text { Cuticle }\end{array}$ & HS8 - Membrane & HS8 - Dendrite & $\begin{array}{c}\text { HS10 - } \\
\text { Surrounding } \\
\text { Cuticle }\end{array}$ \\
\hline $177 \pm 37 \mathrm{MPa}$ & $125 \pm 35 \mathrm{MPa}$ & $64 \pm 14 \mathrm{MPa}$ & $165 \pm 40 \mathrm{MPa}$ \\
\hline
\end{tabular}

4.2.1 Micromechanical properties of the outer membrane at varying indentation depths.

Because the deflection of the outer membrane due to slit compression under loading ultimately triggers the firing of action potentials, we focused on the mechanical properties of the outer membrane. Measurements were taken primarily on slit 8 of HS8, within $1 \mu \mathrm{m}$ of the dendrite tip. Measurements were also taken on slit 7 and slit 6 (to ensure that there are no slit-to-slit differences; data not presented); the results corresponded well with those made on slit 8 . The depth increase of the outer membrane in slits 1-5 made these hardly accessible for AFM. Therefore they were not measured here. However, as discussed in the literature [1,29,40,], this highlights the potentially crucial effects that the depth of the outer membrane (and therefore the curvature) can have on the overall functioning of slit sensilla. 
Figure 8 shows several examples of FDCs taken on the outer membrane with a sharp AFM probe, with lower (15-20 nm, Figs. 8A, B) and higher (25-40 nm, Figs. 8A,B; and 40-50 nm Figs. $8 C, D$ ) penetration depths (note that all curves presented here are shifted along the x-axis for better viewing). In general, the initial portion of the curves shows similar shape and slopes, indicating similar mechanical properties. However, under increased loading (i.e. higher penetration depths) there is a noticeable kink in the $\delta^{3 / 2}$ versus force plots (Figs. $8 \mathrm{~B}, \mathrm{D}$ ). The kink in this case is a decreasing positive slope, indicating a transition from a lower to a higher elastic modulus. The analysis of many whole curves yielded an average elastic modulus of $221 \pm 76 \mathrm{MPa}$.

In order to quantify this change in modulus seen in the higher-penetration FDCs, we have fitted small portions of each curve corresponding to varying penetration depths (Fig. 9). To accomplish this, we pick an isolated portion of the curve and then calculate the modulus from the slope using Eqn. 3, allowing for a shift in the curve along the penetration axis, assuming that if the whole material behaved elastically like this isolated region the line would pass through $(0,0)[41,42]$. When plotting the calculated values of modulus versus penetration depth an "S-shaped" trend is seen; the calculated elastic modulus initially decreases, then increases and levels off (Figs. 9A, B). Because there is data scatter associated with both fitting FDCs as well as from taking FDCs at different locations, Fig. 9A shows the calculated modulus for each FDC which has been translated along the $y$-axis according to the average modulus of the entire curve. In Fig. $9 \mathrm{~A}$ each data set represents calculations made from varying penetration depths for one FDC. All FDCs used in these calculations were then averaged to give the reader a sense of the overall shape of the curves (Fig. 9C). From Fig. 9C it can be seen that there is a concave up portion below $75 \mathrm{nN}$, where the modulus decreases from 225 $\mathrm{MPa}$ to $\sim 122 \mathrm{MPa}$. This concave up shape in the $\delta^{3 / 2}$ vs $F$ curve indicates a viscoelastic material and will be discussed further below. Finally, the modulus appears to reach a limit at $\sim 500 \mathrm{MPa}$, which is much greater than the values measured previously for the epicuticle of the metatarsal pad $[17,25]$.

4.2.2 Membrane micromechanical properties using a large radius probe. We also explored the micromechanical properties of the outer membrane using a large radius 
probe. In order to determine whether such measurements could be taken without the AFM tip touching the sidewalls of the surrounding cuticle we overlaid SEM images onto sectional profiles taken from AFM height images (Fig. 10; scale bars from SEM images and axes from AFM images equivalent). Here it is important to note that the sectional profiles should be taken from raw AFM topographical data (i.e. not flattened) to obtain a more realistic visualization of the tip-surface contact. As Fig. 10 shows, probing is easily achievable with shallow contact angles and no contact between the wider portions of the AFM tips and the walls of the slit.

Figure 11 shows randomly selected FDCs collected on slit 8 of the HS8 organ using a modified tip with a radius of curvature of $408 \mathrm{~nm}$. The analysis of many FDCs $(>30)$ gives an average elastic modulus value of $1930 \pm 590 \mathrm{MPa}$, which is an order of magnitude higher than that obtained with a smaller radius tip. Examination in Hertzian coordinates $\left(\delta^{3 / 2}\right.$ versus the applied force, Fig. 11B) reveals a linear relationship without kinks or significant curvature in the early portions of the curves, indicative of purely elastic behavior. The modulus of several curves was also calculated as a function of penetration depth (Fig. 12; same procedure as that discussed above for Fig. 9). In this case there is no clear trend in behavior, with average-shifted portions (Fig. 12B) all having very similar values well within standard deviation. An average FDC is linear (Fig. 12C), without a clearly viscoelastic portion in the initial penetration region. With the exception of the first fitting region, all values of modulus calculated from this curve range from $\sim 1700 \mathrm{MPa}$ to $\sim 1900 \mathrm{MPa}$.

\section{Discussion}

5.1 Multi-layer structure of outer membrane. The outermost layer of arthropod cuticle, the epicuticle, usually is a very thin structure serving as a protective barrier between the animal and the environment, for example by providing wear resistance and preventing desiccation $[43,44]$. The epicuticle is difficult to isolate from the procuticle, and as a result its mechanical properties have been rarely investigated [43]. It is a layered structure which includes cement, oriented lipid, and cuticulin layers $[1,39,45]$. Our 
results point to the layered nature of the epicuticle, with a few discernable layers (or regions of varying mechanical properties) (see Figs. 7, 9 and 12). When using a very soft cantilever (spring constant $\sim 0.2-0.4 \mathrm{~N} / \mathrm{m}$; Fig. 7, Table 1) with low indentation depths $(\sim 5 \mathrm{~nm})$ we see a very compliant surface with a modulus of $125 \mathrm{MPa}$. As the indentation depth increases to $40 \mathrm{~nm}$ (Fig. 9) when probing deeper into the subsurface, the apparent elastic modulus increases to $\sim 500 \mathrm{MPa}$.

During nano-indentation experiments, given a constant penetration depth, the stress field directly beneath the probe increases with increasing tip radius. Therefore probing with a large radius tip such as that shown in Fig. 10 or Fig. 3B inset, results in the interaction with materials much further below the surface than can be achieved with a sharp probe. Here we obtain modulus values which are much higher than recorded in measurements using a large radius probe. This is interesting because the outer membrane layer that spans the slit sensillum is very thin, on the order of $\sim 200 \mathrm{~nm}$. It is unlikely that the viscous receptor lymph below the outer membrane is causing such a dramatic increase in modulus. However, electron micrographs (see Fig. 10 in ref. [29]) show random, residual nanoribbon-like structures which span the slit below the outer membrane. There also appears to be a thin layer of greater electron density at the inside end of the outer membrane. The tensile modulus of single chitin fibrils is estimated to be as high as 10 s to 100 s of GPa $[46,47]$. Our present results suggest that there is some underlying layer of chitin fibrillar structure or some cross-linked protein that serves to support the outer membrane during the formation of the slit sensors. A stiff underlying layer suggested here would not necessarily heavily affect the properties of the slit sensor because such fibrils are not resistant to deflections perpendicular to their long axis resulting from buckling under compressive loading (i.e. their high strength only applies to tensile loading).

We propose that the high modulus calculated using larger penetration depths and large radius probes, are occurring as the stress field begins to interact with this fibril layer. As shown by the example in Fig. 9, the modulus increases to $\sim 500 \mathrm{MPa}$ as the indentation depth increases. However, the larger probe allows us to apply much larger forces without risking plastic deformation. Under large loads $(\sim 4 \mu \mathrm{N}$ maximum load presented 
in Fig.11), the deformational behavior of the tip-membrane contact interaction transitions from purely surface deformation to a combination of surface deformation with some tensile stretching of the membrane itself. We then observe the dramatic increase in elastic modulus as a result of the large tensile modulus of cross-linked fibrils. At this time, it is unclear if a stiff underlying layer serves any structural function.

5.2 Consequences of viscoelastic outer membrane layer. Mechanosensory organs in arthropods have adaptive, time-dependent behavior $[48,49,50]$. The number of action potential impulses fired by the sensory organ as a function of time, $y(t)$, can often be described by a simple power law [1,51]:

$$
y(t)=a \cdot d \cdot t^{-k}
$$

where $a$ is an amplification constant, $d$ is the stimulus amplitude, $t$ is time, and $k$ is a constant describing how quickly the receptor response declines under a constant stimulus. In the case of slit sensilla, $k$ ranges from 0.2 to 0.7 . Whereas $k=0$ corresponds to a frequency-independent displacement detector, $k=1$ indicates a pure first-order velocity detector.

The response behavior of slit sensilla is somewhere between these two extreme cases and can be considered in terms of time-dependent properties of materials [1,51]. Recently, we have described the time- and temperature-dependent properties of the thick epicuticular layer on the metatarsal pad transferring mechanical stimuli to the HS10 lyriform organ, using the so-called creep compliance function [25,52]. This function produces a sigmoidal curve which describes the mechanical response to a statically applied load and the material's transition from the glassy regime (higher apparent elastic modulus) to the rubbery regime (lower apparent elastic modulus). According to this approach, the apparent elastic modulus is proportional to $e^{-t / \tau}$, where $t$ is time and $\tau$ is the relaxation time, which corresponds to the point of inflection on the sigmoidal curve. The relaxation time of a viscoelastic material is a function of its dynamic viscosity, and as a result is strongly dependent on temperature. In our previous studies we observed material relaxation times ranging from $\sim 0.01 \mathrm{~s}$ at slightly elevated temperatures $\left(35^{\circ} \mathrm{C}\right)$ to $\sim 5 \mathrm{~s}$ at lower temperatures $\left(19^{\circ} \mathrm{C}\right)$. Using our previously 
described fitting procedure [25,52], we found that the initial viscoelastic portion of the curves shown in Fig. 8 exhibit average relaxation times of $\sim 0.2-0.4 \mathrm{~s}$, which is in good agreement with our previous results at $22-24^{\circ} \mathrm{C}$.

The electrophysiological response of HS8 sensory neurons to loading as reported in the literature [51] shows a sharp decrease in the impulse rate, $y(t)$, within the first few seconds of load application. To see how the creep compliance function changes as a function of time, we have plotted $1-e^{-t / \tau}$ versus time in Fig. 13 ("dimensionless creep compliance") for several values of $\tau$. Also listed is the approximate temperature at which the specific $\tau$ value of the epicuticle applies. It should be noted that the true creep compliance function will scale differently along the $y$-axis, but the shape along the $x$-axis is preserved (on a log scale). From this plots one can see that the transition from higher modulus (lower limit, see refs. [25,52]) to lower modulus (upper limit) in response to a statically applied load occurs on the same order of time as the decrease in $y(t)$ for HS8 reported by Bohnenberger [51]. Because $\tau$ varies with temperature, we suggest that the time-dependent sensory response described by Eqn. 6 should be dependent on temperature, and may be represented by including a temperature-dependent rate constant:

$$
y(t, T)=a \cdot d \cdot t^{-k(T)}
$$

For comparison we have also plotted $t^{-k}$ versus time for several $k$ values, with each data set being normalized to $0.001^{-k}$. The time required for a given percentage decrease in $t^{-k}$ decreases as $k$ decreases. On the other hand, the transitional time between upper and lower limits according to the creep compliance function decreases with decreasing $\tau$. Perhaps then the rate constant $k$ is inversely related to $\tau$. However, this analysis is oversimplified in that it only considers the viscoelastic mechanical properties of the outer membrane. In reality, several other viscous components also contribute to the time-dependent sensory adaptation of the organ's neural response. These components include the receptor lymph, the dendrite and its sheath and the coupling cylinder, to name a few and may have to be considered in addition to the signaling properties of the neuron itself. 


\section{Conclusion}

In conclusion, we characterized micromechanical properties of two strain-sensitive lyriform organs of the wandering spider Cupiennius salei by direct force measurement. Using AFM nanoindentation approaches we were able to quantify differences in the mechanical properties of the top-most surface layers of the cuticle immediately surrounding the slits, of the outer slit membrane, and the small dendrite ending in it. Our measurements also indicated layered membrane structure with increasing modulus. Finally, we also observed a viscoelastic response in the initial portions of the FDCs measured at moderate indentation depths. Simple analysis of the viscoelastic portion of the penetration curves yielded relaxation times on the order of 0.4 seconds. These relaxation times are in the range of the time it takes to observe a drastic decrease in the rate at which impulses in the HS8 organ fire in response to a static load.

Previous experimental modeling studies have only considered the lyriform organs as holes in a linearly elastic material with a modulus of $18 \mathrm{GPa}$ [16], which is orders of magnitude higher in modulus than what we have observed here. However, the complex layered structure and viscoelastic behavior described within this study could have unforeseen effects on how the lyriform organ deforms in response to compressional loading. Therefore to better understand the function of the lyriform organs further experimentation must consider the viscoelastic nature of the cuticle material. As a starting point, the bending behavior of the outer membrane, which is ultimately responsible for deforming the dendrite, has not been examined. Modeling experiments could incorporate the mechanical properties we obtained by directly probing the membrane here to examine the buckling behavior of a multilayered, suspended membrane with some viscoelastic component. This result reflects the biomechanical processes associated with this sensory organ in contrast to previous studies which only considered the compression of cracks as an elastic solid. Modeling experiments can be setup using the viscoelastic mechanical properties of the membrane determined from 
this study to estimate the time- and temperature-dependent deformational behavior of the slit systems and the suspended outer membrane.

Acknowledgements: Financial support came from the National Science Foundation, Division of Materials Research, DMR-1002810 and World Materials Network Award DMR-1209332. We are grateful to the Department for Neurobiology of the University of Vienna for providing the spiders and Peter Fratzl, MPI for helpful discussions. 


\section{Figures}
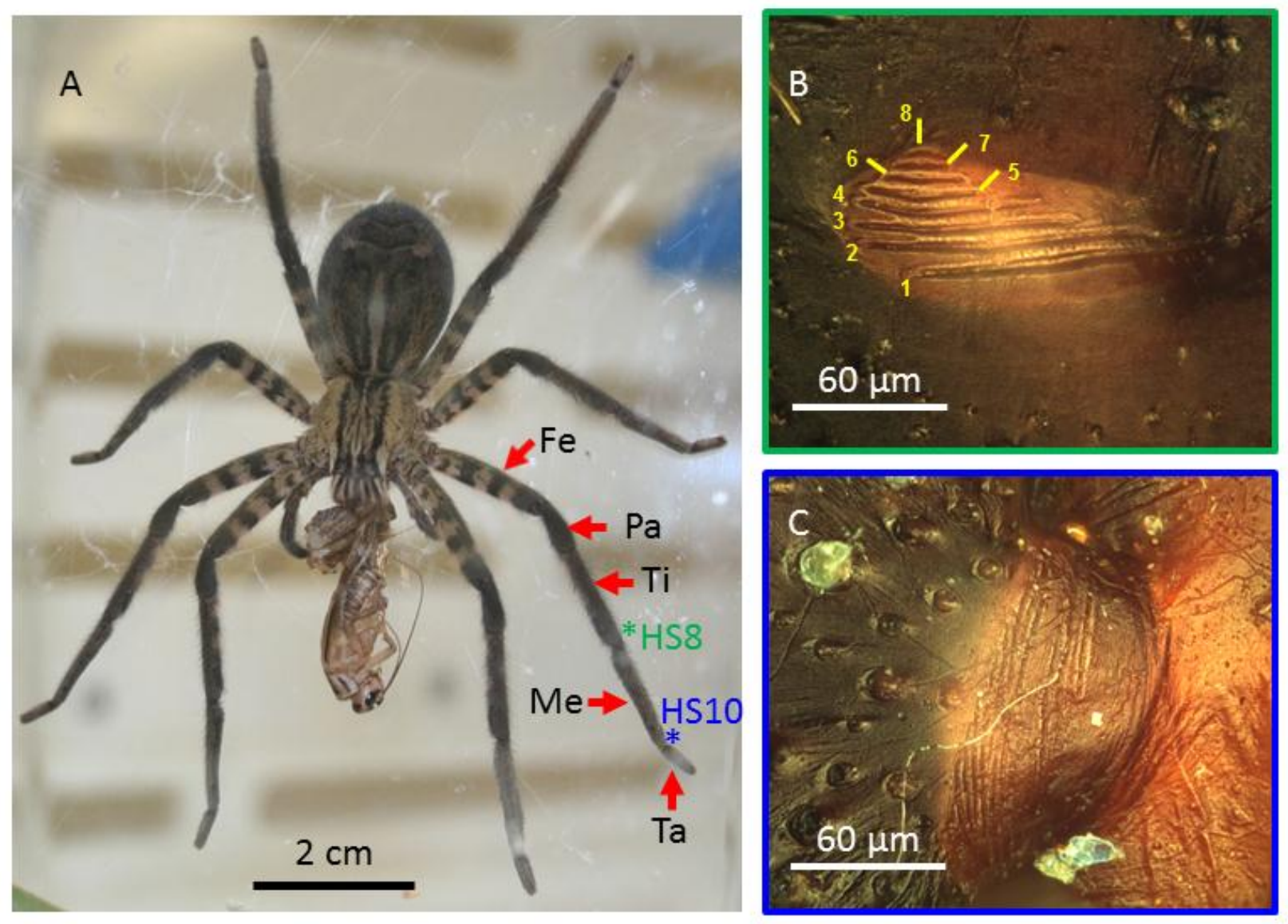

Figure 1: (A) Adult female Cupiennius salei. Arrows demark different leg segments: Ta = tarsus, $\mathrm{Me}=$ metatarsus, $\mathrm{Ti}=$ tibia, $\mathrm{Pa}=$ patella, $\mathrm{Fe}=$ femur. Colored asterisks demark approximate location of two lyriform organs examined in this study: green = HS8, located on posterior side of the leg; blue = HS10, located on the dorsal side of the leg. (B) Optical image of HS8 lyriform organ, slit numbering here is used for reference in later results. (C) Optical image of HS10 lyriform organ. 


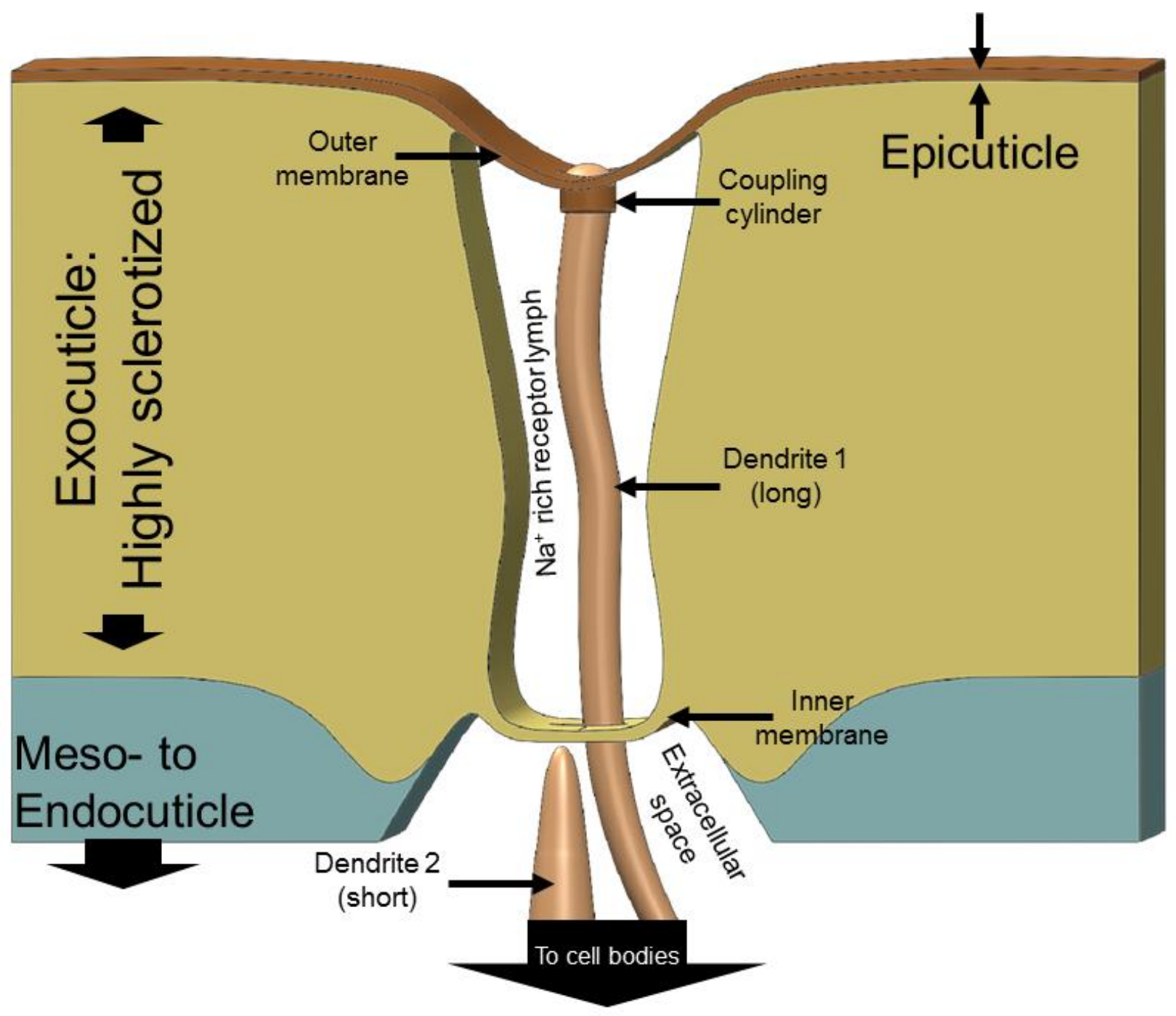

Figure 2: Schematic depicting a section through a single slit sensillum [1,29]. Not shown here is a sheath which covers both dendrites, extending from the coupling cylinder down to the cell bodies. 


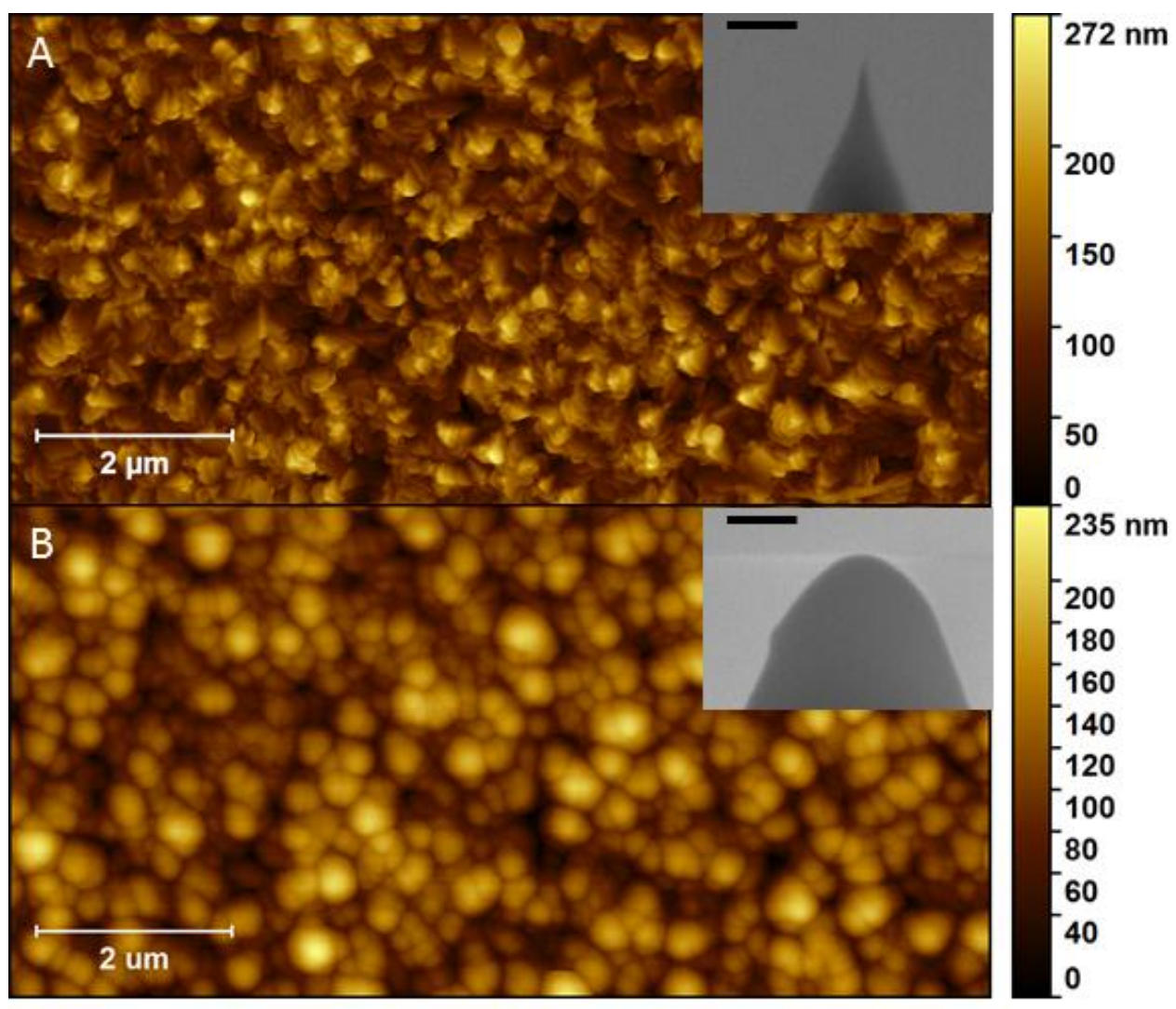

Figure 3: AFM height images of standard $\mathrm{TiO}_{2}$ roughness samples used to determine tip shape by deconvolution (A) before and (B) after oxidation at $1100^{\circ} \mathrm{C}$ in air for 12 hours. Insets are SEM images of each tip used to take the AFM image, scale bar $=300 \mathrm{~nm}$. 

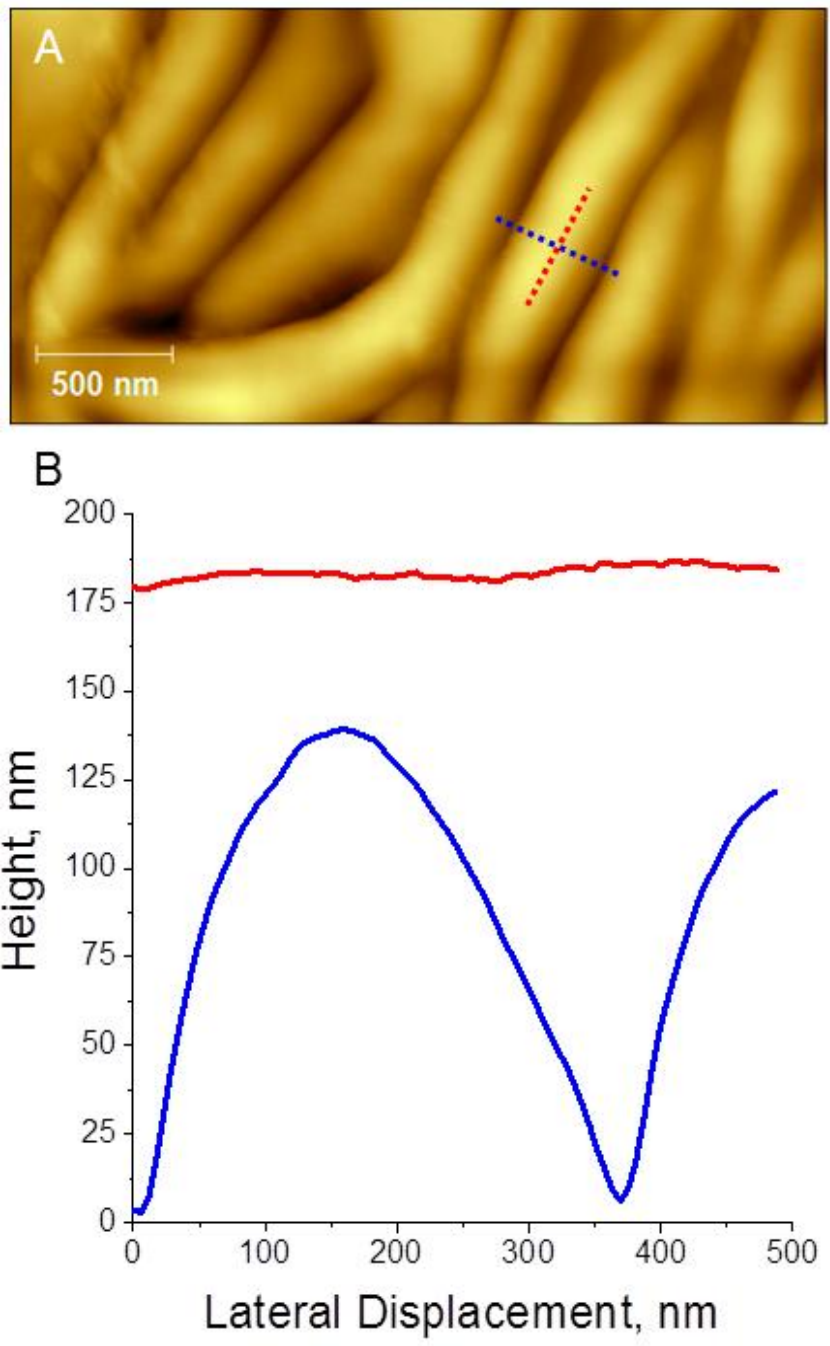

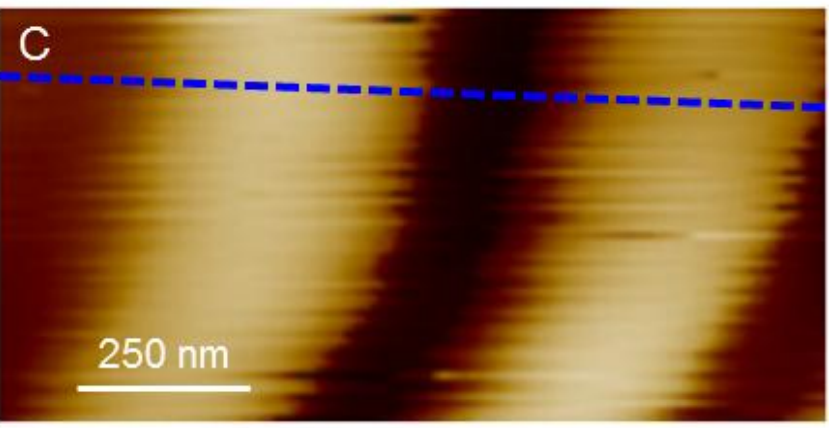

D

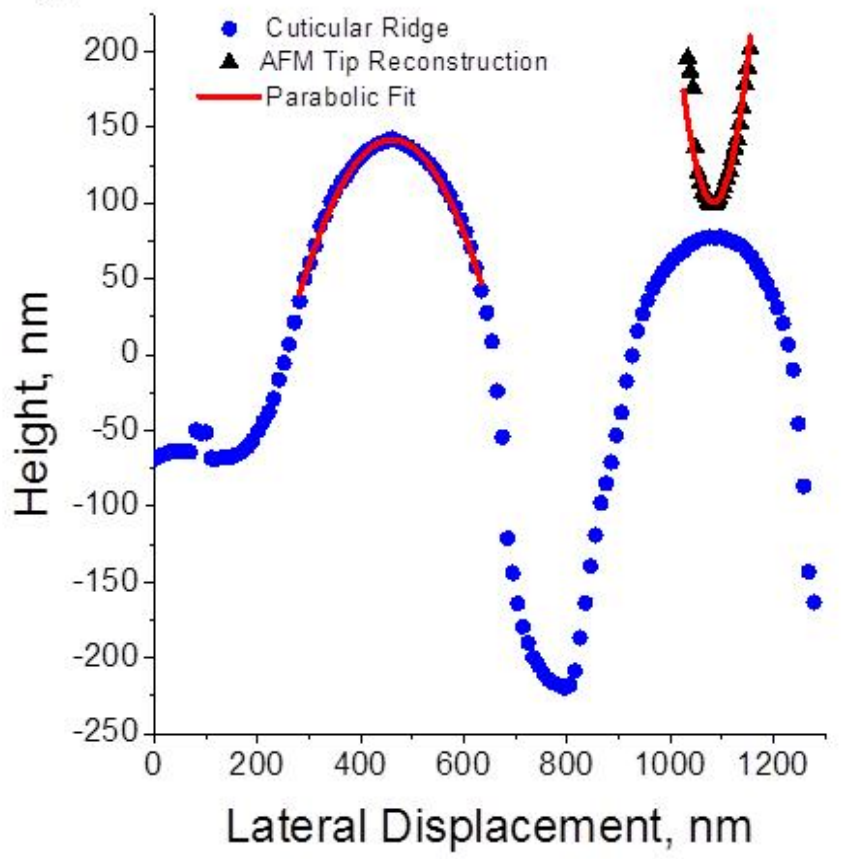

Figure 4: (A) High resolution (1024 pixels $\times 512$ pixels) AFM topography image of epicuticular ridges found on the surface of the cuticular bridges separating neighboring slits of HS10. (B) Profiles along the colored dashed lines in $\mathrm{A}$, showing that at the ridge peak one can assume the shape of a paraboliccylinder. (C) Height image obtained from high-resolution SFS mapping (128 pixels x 64 pixels) of ridges on the cuticle surrounding HS10 slits. (D) Profile obtained from (C), fit with fitting by a parabolic function to determine radius of curvature. 

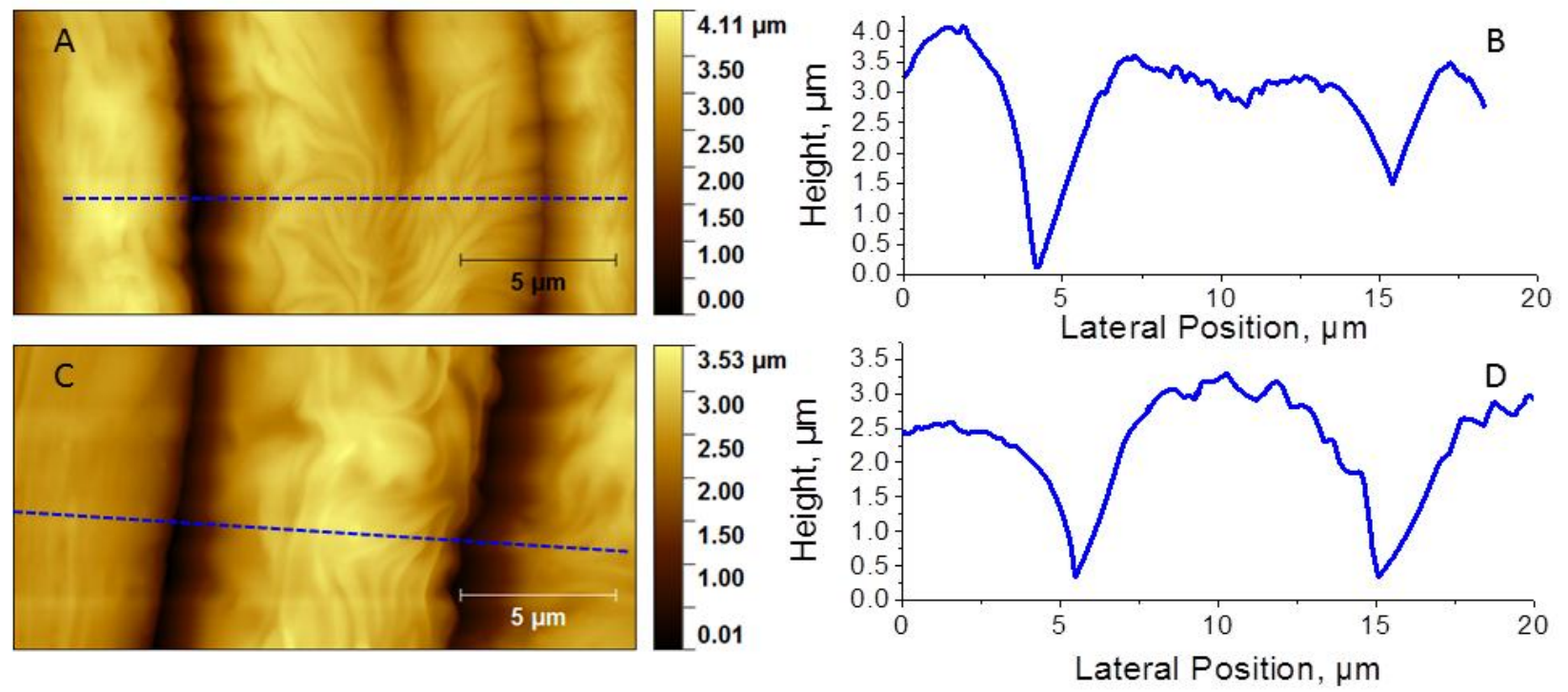

Figure 5 AFM topography images of slits located in the metatarsal lyriform organ (HS10). (B,D) Profiles along the blue dashed lines in $A$ and $C$, respectively. 

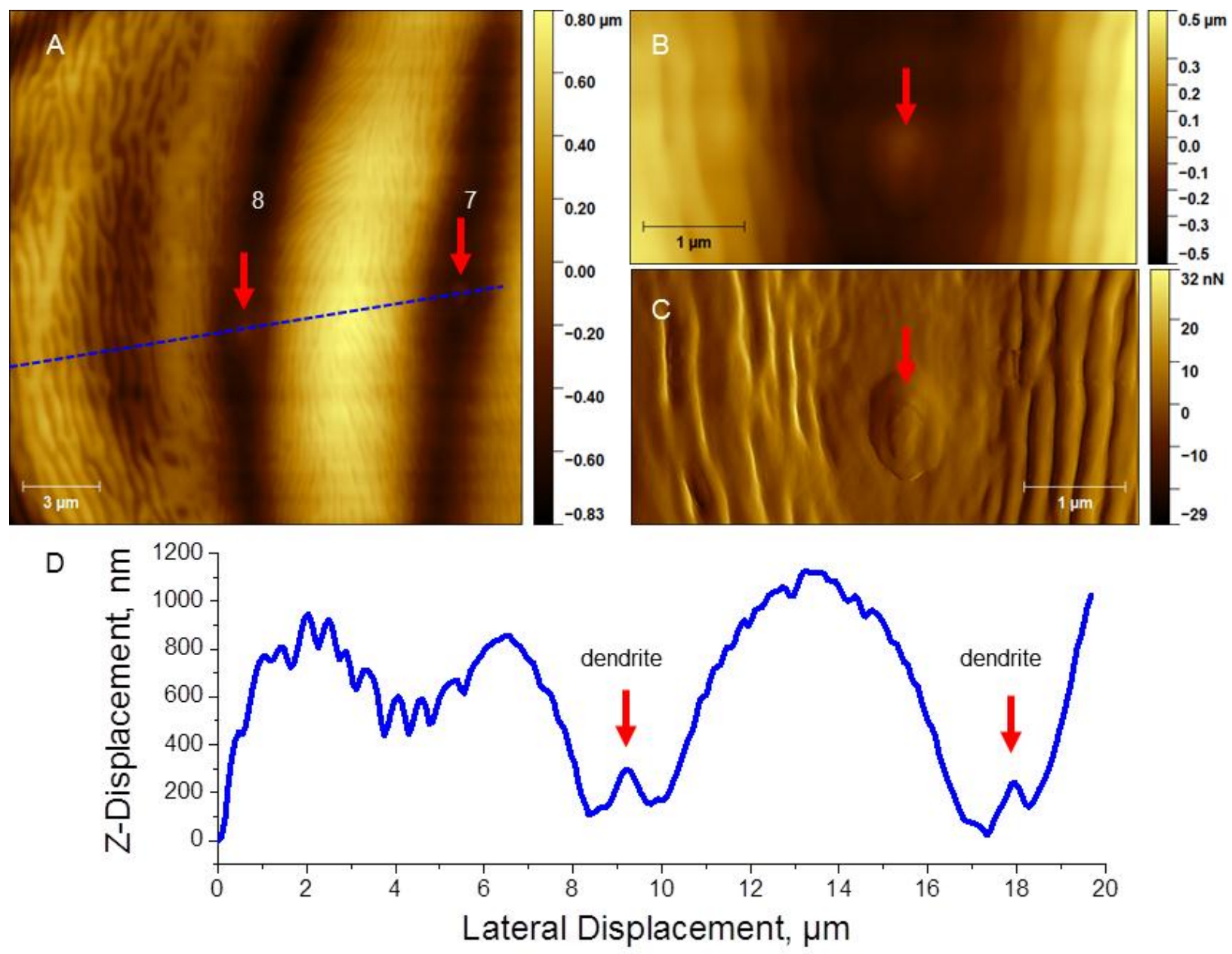

Figure 6: (A) AFM topography image containing a portion of slits 7 and 8 of the HS8 lyriform organ, in which the slit width and shallowness of the covering membrane allow measurements of the outer membrane as well as the dendrite region. A zoomed-in height image $(B)$ and error image $(C)$ of the dendrite region. The error image can be viewed as a type of differential height image, and as such is presented because it gives clear outlines in area where height changes significantly. Here the error image aids in visualizing the dendrite region. (D) Profile taken from (A) along the blue dotted line. Red arrows indicate dendrite regions. 

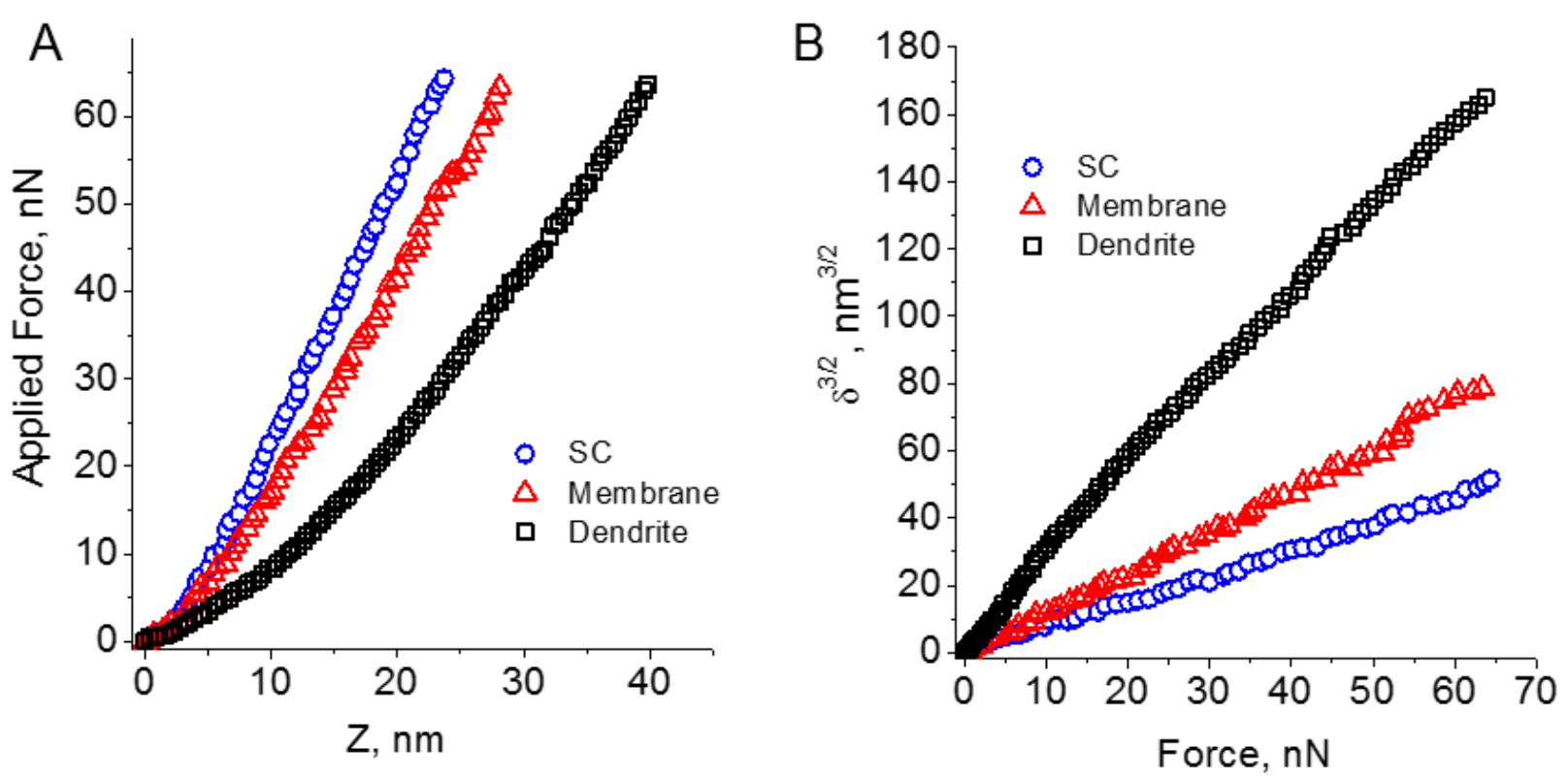

Figure 7: Example force versus displacement $(A)$ and penetration depth versus force $(B)$ plots from three distinct regions of HS8 lyriform organ on a freshly autotomized leg. 

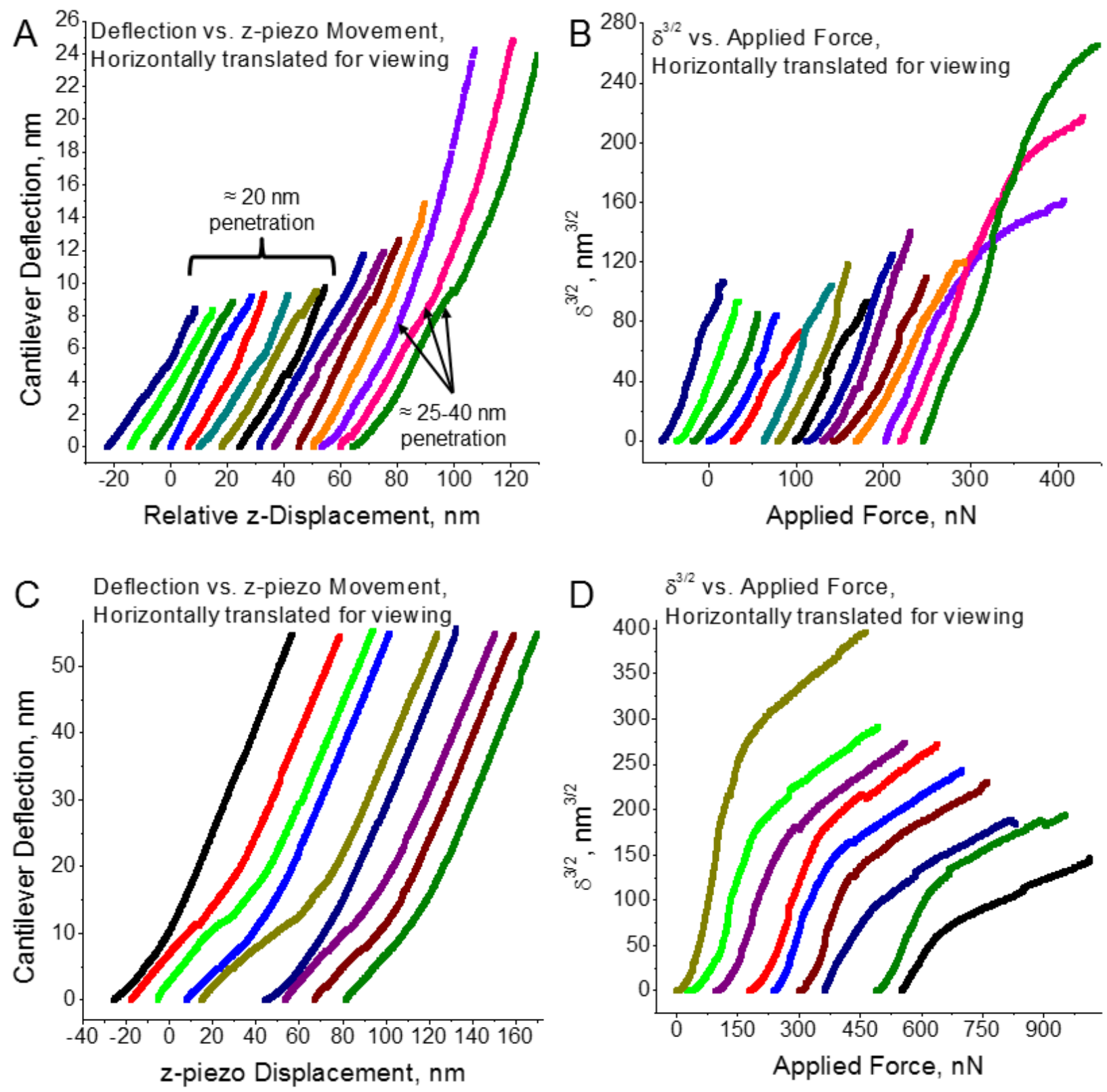

Figure 8: Example cantilever deflection versus displacement $(A, C)$ and penetration depth versus force $(B, D)$ plots obtained from loading curves taken from an HS8 lyriform organ on a rehydrated leg using low to moderate $(A, B)$ and higher $(C, D)$ deflections thresholds, resulting in applied forces ranging from $\sim 64 \mathrm{nN}$ to $\sim 400 \mathrm{nN}$. 


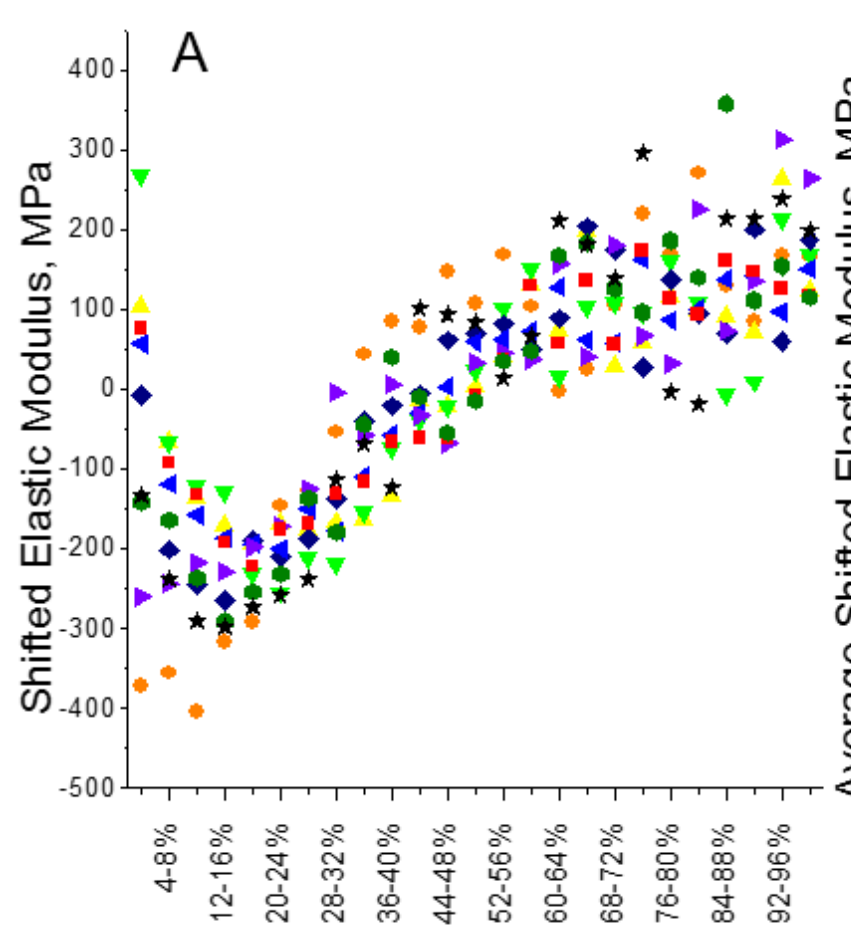

Segment of FDC Used for Calculation

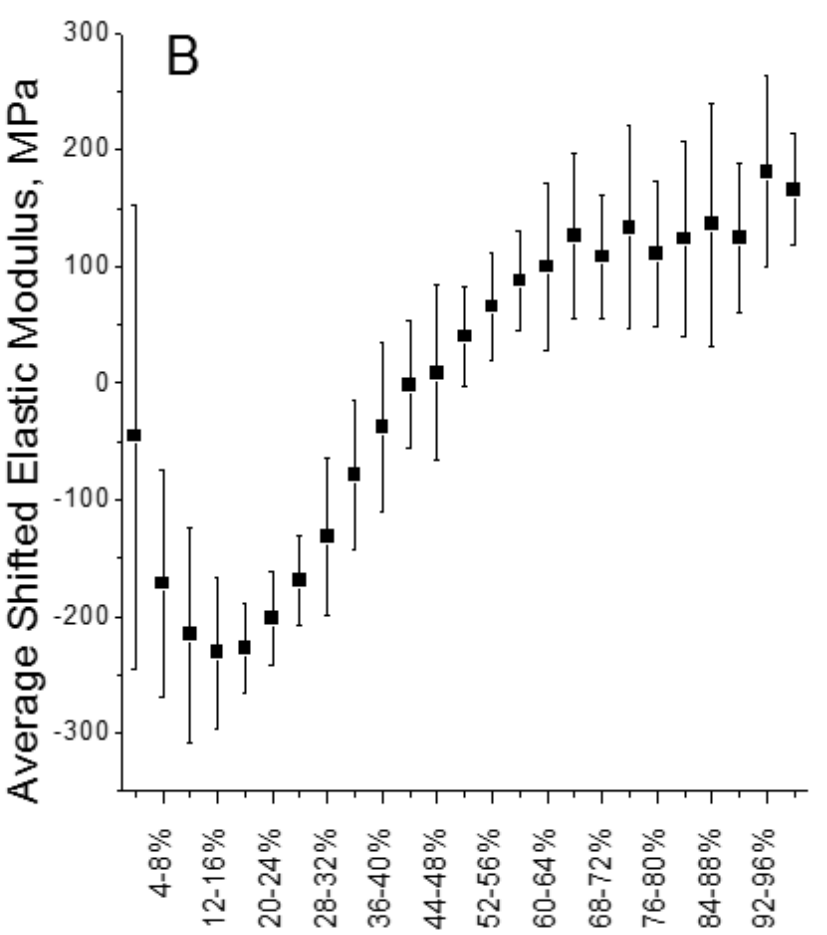

Segment of FDC Used for Calculation

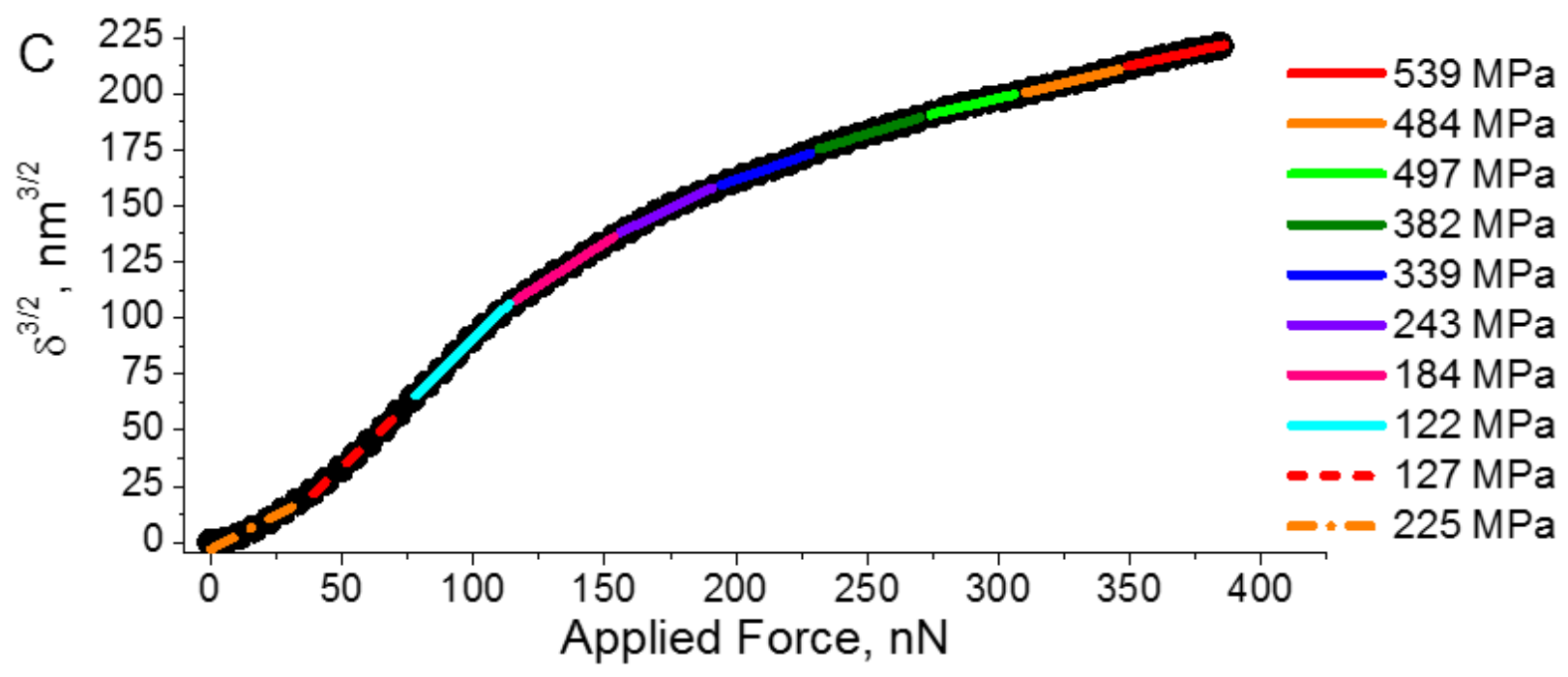

Figure 9: (A) Elastic modulus values calculated from different portions of 9 separate FDCs obtained with high ( $400 \mathrm{nN}$ ) applied forces. Here each set of data represents a 4\% interval of the total deflection. For example, when the total deflection is $50 \mathrm{~nm}$, then each group of data represents the modulus calculated from $2 \mathrm{~nm}(\sim 15 \mathrm{nN})$ segments of the FDC. (B) Average modulus values from all 9 curves, error bars represent one standard deviation. (C) Representative FDC formed from the average of all 9 deflection curves shown in Fig. $7 \mathrm{C}$. Here each $10 \%$ interval was fit for modulus and overlaid on the plot to give the reader a sense of how the slope of the curve is changing with applied force (slope directly proportional to calculated elastic modulus). Legend indicates elastic modulus value calculated from each fit segments (color copy can be obtained online). 


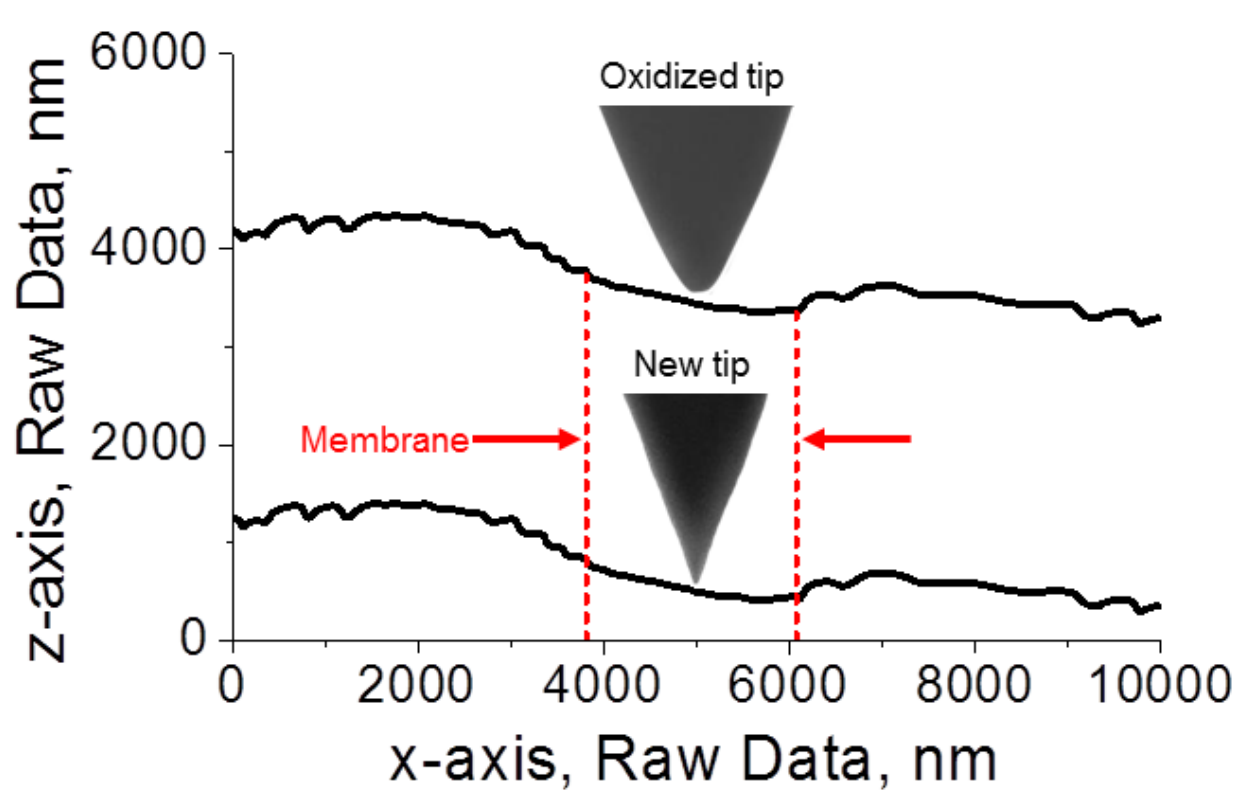

Figure 10: SEM images of a new AFM tip (lower part of graph) and an oxidized AFM tip (upper part of graph) above a line profile of unprocessed AFM height sensor data. Graph and SEM images have been sized such that the z-axis, x-axis, and SEM scale bars (cropped out) are all equal to give the reader an impression of the relative size of the slit-tip interaction. Here it can be seen that FDCs with indentation depths $>100 \mathrm{~nm}$ can easily be performed on the slit outer membrane without the AFM tip contacting the slit walls (demarked in red dashed line and arrows).
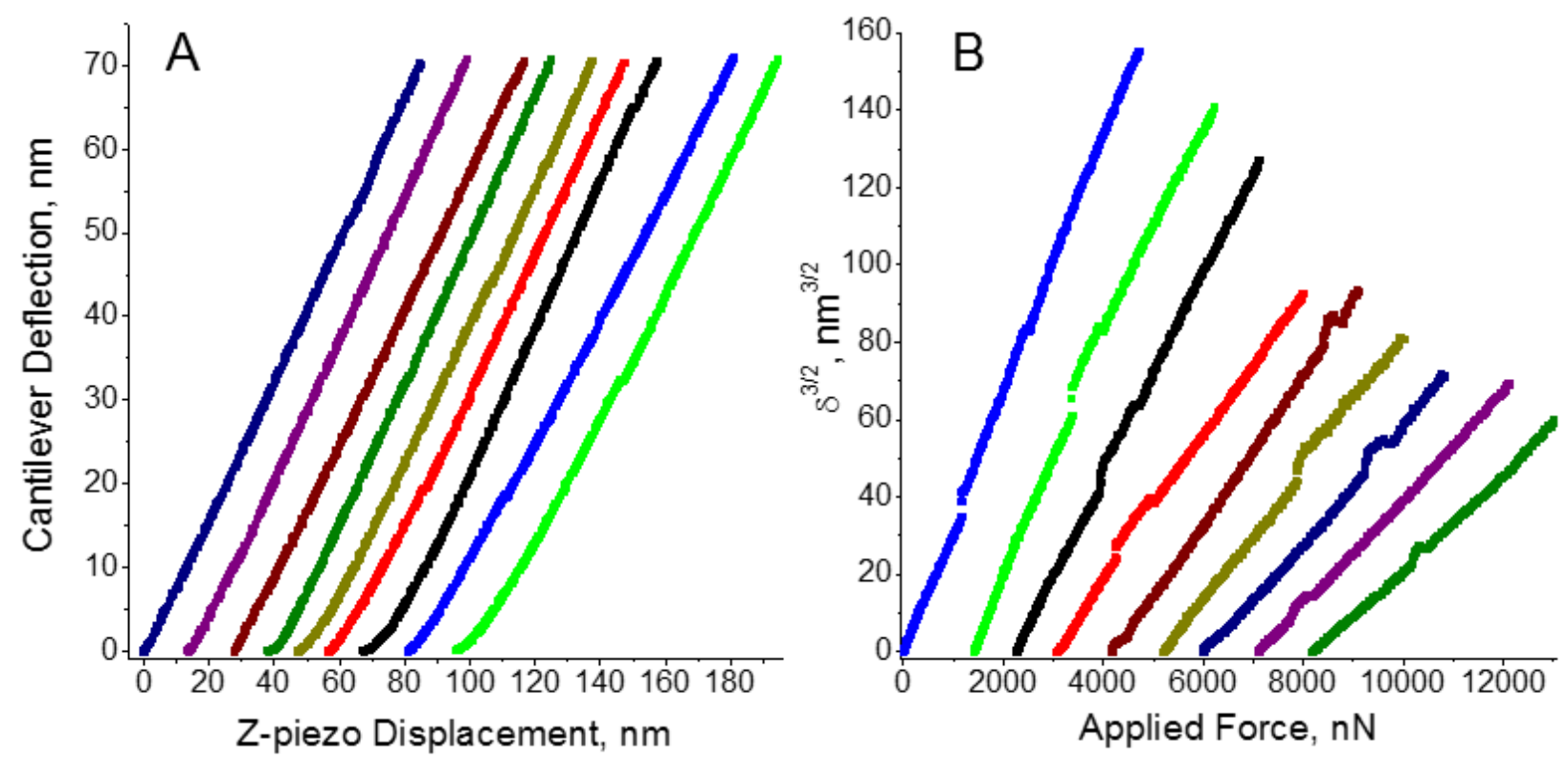

Figure 11: Example cantilever deflection versus displacement $(A)$ and penetration depth versus force (B) plots obtained from loading curves taken on an HS8 lyriform organ using a modified tip with a radius of 
curvature of $408 \mathrm{~nm}$. Similar to Fig. 8, these curves have been horizontally translated for better viewing. Curve colors in A correspond to those in B. Color copy can be obtained online.

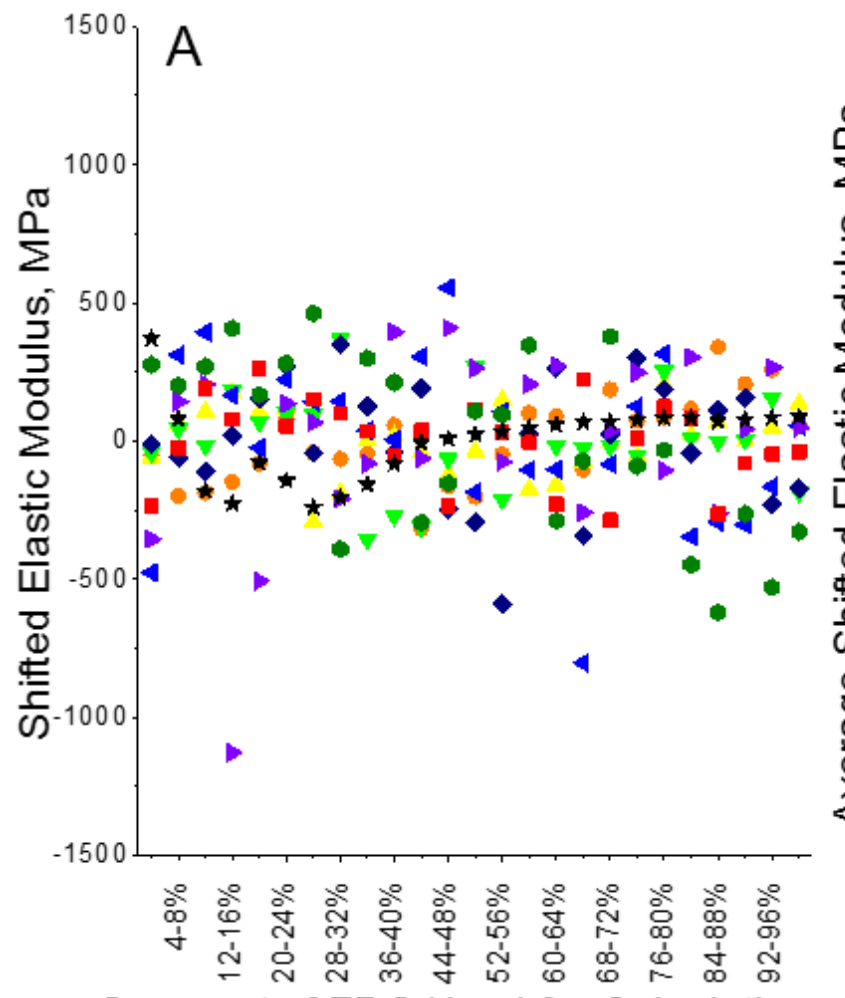

Segment of FDC Used for Calculation

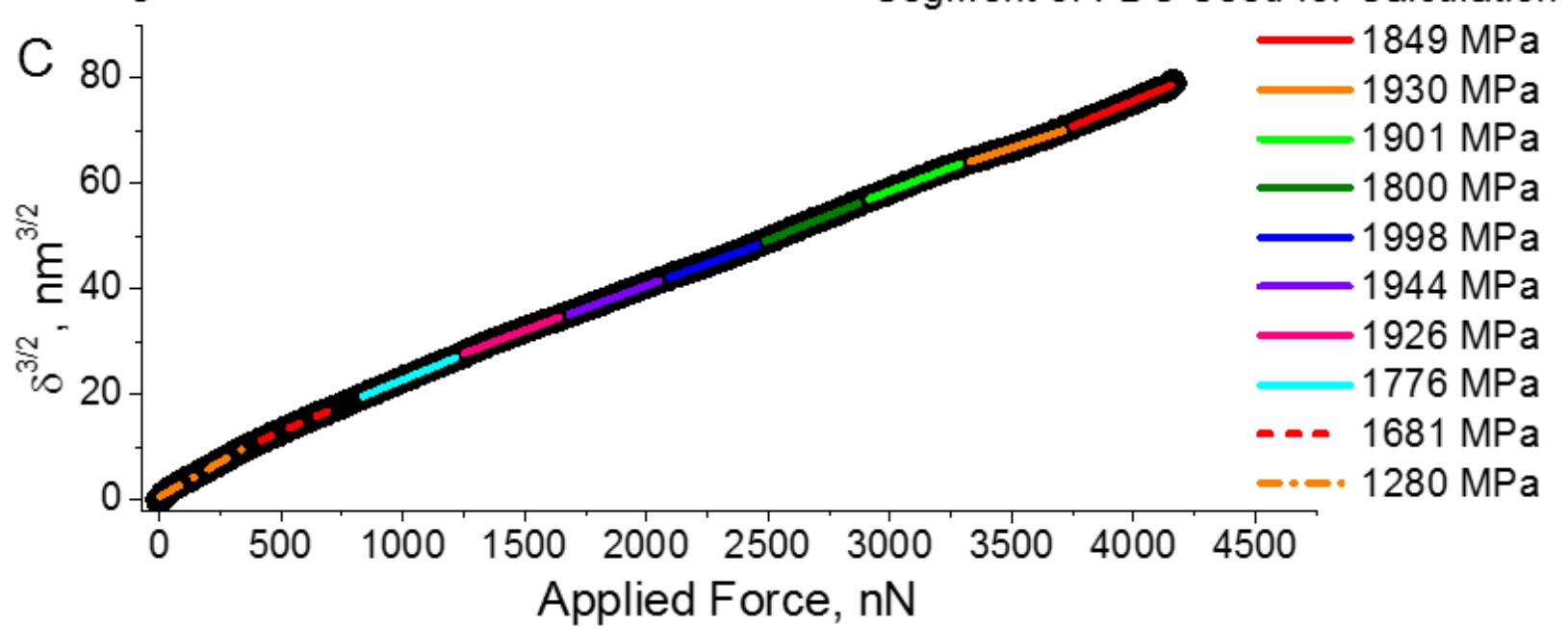

Figure 12: (A) Elastic modulus values calculated from different portions of 9 separate FDCs obtained with a modified AFM tip (408 nm radius of curvature). Each set of data represents a $4 \%$ interval of the total deflection. (B) Average modulus values from all 9 curves, error bars represent one standard 
deviation. (C) Representative FDC formed from the average of all 9 deflection curves shown in Fig. 11A, with calculated modulus values from 10\% interval regions (color copy can be obtained online).
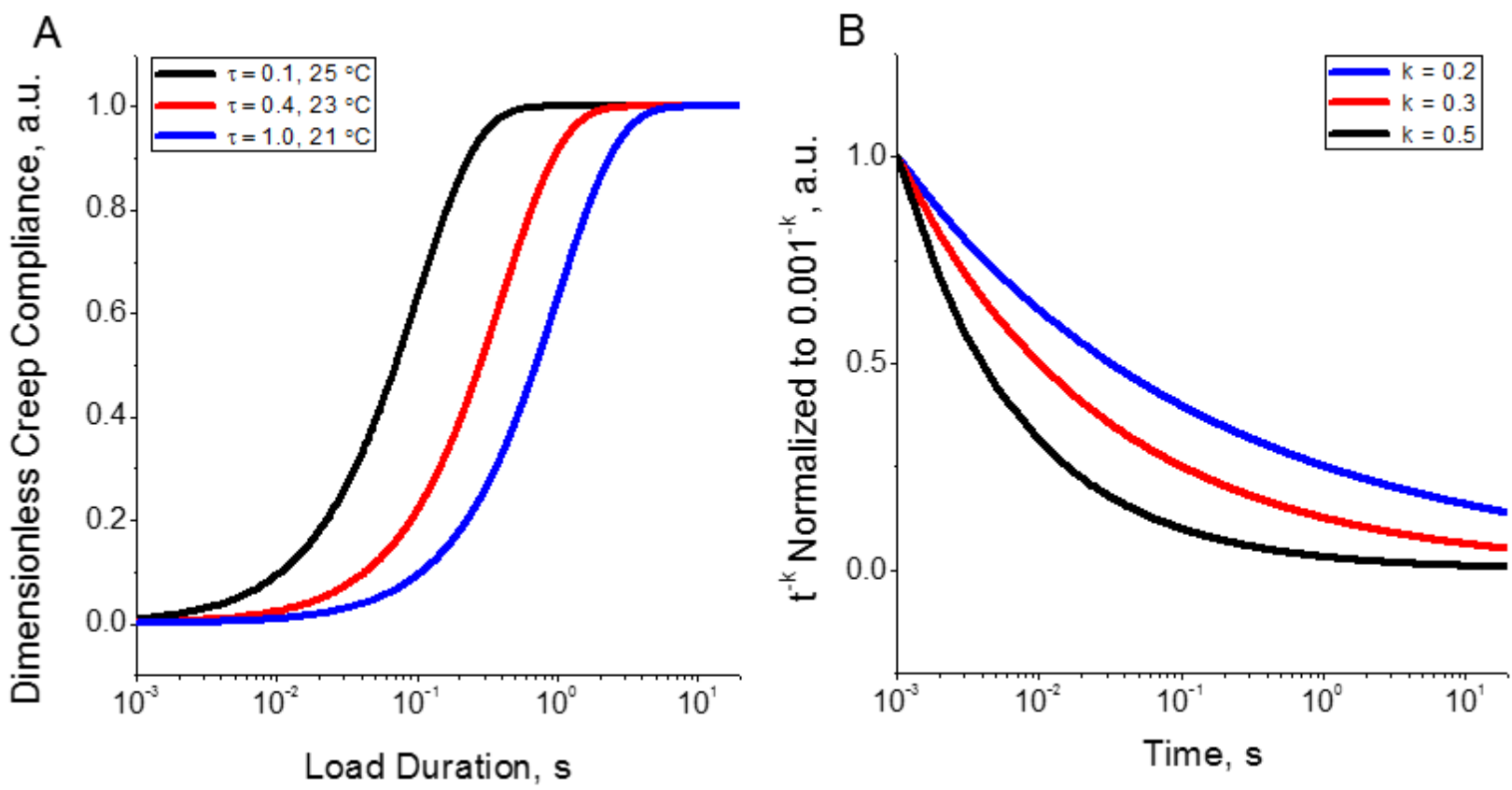

Figure 13: (A) "Dimensionless creep compliance function" (see text) for relaxation times of $0.1 \mathrm{~s}, 0.4 \mathrm{~s}$, and $1.0 \mathrm{~s}$. Approximate temperature in which the spider epicuticle would exhibit these relaxation times included in legend. (B) Plot of the exponential function $\boldsymbol{t}^{-\boldsymbol{k}}$ for $\boldsymbol{k}=0.2,0.3$, and 0.5 normalized to the maximum value of each set, which is $\mathbf{0 . 0 0 1}{ }^{-\boldsymbol{k}}$ in the range we have plotted here. 


\section{References}

[1] Barth FG. A Spider's World: Senses and Behavior. Heidelberg: Springer-Verlag; 2002.

[2] Albert J, Friedrich O, Dechant HE, Barth FG. Arthropod touch reception: spider hair sensilla as rapid touch detectors. J. Comp. Physiol. A 2001;187:303-12.

[3] Dechant HE, Rammerstorfer F, Barth FG. Arthropod touch reception: stimulus transformation and finite element model of spider tactile hairs. J. Comp. Physiol. A 2001;187:313-22.

[4] Fratzl P, Barth FG. Biomaterial systems for mechanosensing and actuation. Nature 2009;462:442-8.

[5] Barth FG. How To Catch the Wind: Spider Hairs Specialized for Sensing the Movement of Air. Naturwissenschaften 2000;87:51-8.

[6] Barth FG. Spider mechanoreceptors. Current Opinion in Neurobiology 2004;14:415-22.

[7] McConney ME, Schaber CF, Julian MD, Eberhardt WC, Humphrey JAC, Barth FG, Tsukruk VV. Surface force spectroscopic point load measurements and viscoelastic modelling of the micromechanical properties of air flow sensitive hairs of a spider (Cupiennius salel). J. R. Soc. Interface 2009;6:681-94.

[8] Klopsch C, Kuhlmann HC, Barth FG. Airflow elicits a spider's jump towards airborne prey. I. Airflow around a flying blowfly. J. R. Soc. Interface 2012;9:2591-602.

[9] Barth FG, Pickelmann P. Lyriform slit sense organs. J. Comp. Physiol. 1975;103:39-54.

[10] Barth FG, Stagl J. The slit sense organs of arachnids. Zoomorphologie 1976;86:1-23.

[11] Barth FG. Slit Sensilla and the Measurement of Cuticular Strains. In: Barth FG, editor. Neurobiology of Arachnids: Springer Berlin Heidelberg; 1985. p. 162-88.

[12] Barth FG. Spider senses - technical perfection and biology. Zoology 2002;105:271-85.

[13] Barth FG, Libera W. Ein Atlas der Spaltsinnesorgane von Cupiennius salei Keys. Chelicerata (Araneae). Z Morph Tiere 1970;68:343-69.

[14] Barth FG, Geethabali. Spider vibration receptors: Threshold curves of individual slits in the metatarsal lyriform organ. J. Comp. Physiol. 1982;148:175-85.

[15] Barth FG, Ficker E, Federle H-U. Model studies on the mechanical significance of grouping in compound spider slit sensilla (Chelicerata, Araneida). Zoomorphology 1984;104:204-15.

[16] HößI B, Böhm HJ, Rammerstorfer FG, Barth FG. Finite element modeling of arachnid slit sensilla- I. The mechanical significance of different slit arrays. J. Comp. Physiol. A 2007;193:445-59.

[17] McConney ME, Schaber CF, Julian MD, Barth FG, Tsukruk VV. Viscoelastic nanoscale properties of cuticle contribute to the high-pass properties of spider vibration receptor (Cupiennius salei Keys). J. R. Soc. Interface 2007;4:1135-43.

[18] HößI B, Böhm HJ, Schaber CF, Rammerstorfer FG, Barth FG. Finite element modeling of arachnid slit sensilla: II. Actual lyriform organs and the face deformations of the individual slits. J. Comp. Physiol. A 2009;195:881-94.

[19] Skordos A, Chan PH, Vincent JFV, Jeronimidis G. A novel strain sensor based on the campaniform sensillum of insects. Phil. Trans. A 2002 360:239-253. 
[20] Calvert P. Embedded mechanical sensors in artificial and biological systems, in: FG Barth, JAC Humphrey, TW Secomb (Eds), Sensors and Sensing in Biology and Engineering, Springer-Verlag ,Wien, 2003.

[21] Vincent JFV, Clift SE, Menon C. Biomimetics of Campaniform Sensilla: Measuring Strain from the Deformation of Holes. J. Bionic Eng. 2007;4: 63-76.

[22] Summerer L. Thinking tomorrows' space - Research trends of the ESA advanced concepts team 2002-2012. Acta Astronautica 2014;95:242-59.

[23] Kang D, Pikhitsa PV, Choi YW, Lee C, Shin SS, Piao L, Park B, Suh K-Y, Choi, M. Ultrasensitive mechanical crack-based sensor inspired by the spider sensory system. Nature 2014;516:222-6.

[24] Fratzl P. Applied physics: The virtues of tiling. Nature 2014;516:178-9.

[25] Young SL, Chyasnavichyus M, Erko M, Barth FG, Fratzl P, Zlotnikov I, Politi Y, Tsukruk, VV. A spider's biological vibration filter: Micromechanical characteristics of a biomaterial surface. Acta Biomaterialia 2014;10:4832-42.

[26] Erko M, Younes-Metzler O, Rack A, Zaslansky P, Young SL, Milliron G, Chyasnavichyus M, Barth FG, FratzI P, Tsukruk VV, Zlotnikov I, Politi Y. Micro- and nano-structural details of a spider's filter for substrate vibrations: relevance for low-frequency signal transmission. J. R. Soc. Interface 2015;12.

[27] Molina J, Schaber CF, Barth FG. In search of differences between the two types of sensory cells innervating spider slit sensilla (Cupiennius salei Keys.). J. Comp. Physiol. A 2009;195:1031-41.

[28] Rick R, Barth F, Pawel A. X-ray microanalysis of receptor lymph in a cuticular arthropod sensillum. J. Comp. Physiol. 1976;110:89-95.

[29] Barth F. Die Physiologie der Spaltsinnesorgane. J. Comp. Physiol. A 1972;78:315-36.

[30] Barth F. Die Physiologie der Spaltsinnesorgane. J. Comp. Physiol. B 1972;81:159-86

[31] Maier L, Root T, Seyfarth E-A. Heterogeneity of spider leg muscle: Histochemistry and electrophysiology of identified fibers in the claw levator. J Comp Physiol B 1987;157:28594.

[32] Höger U, Torkkeli PH, Seyfarth E-A, French AS. Ionic Selectivity of Mechanically Activated Channels in Spider Mechanoreceptor Neurons. J. Neurophysiol. 1997;78:2079-85.

[33] Tsukruk VV, Singamaneni SS. Scanning Probe Microscopy of Soft Matter: Fundamentals and Practices. Wiley-VCH ; Weinheim; 2011

[34] Cook S, Schaffer TE, Chynoweth KM, Wigton M, Simmonds RW, Lang KM. Practical implementation of dynamic methods for measuring atomic force microscope cantilever spring constants. Nanotechnology 2006;17:2135-45.

[35] Sneddon IN. The relation between load and penetration in the axisymmetric Boussinesq problem for a punch of arbitrary profile. Int. J. Eng. Sci. 1965;3:47-57.

[36] Johnson KL. Contact Mechanics. Cambridge University Press: Cambridge Cambridgeshire; 1985.

[37] Boresi AP. Advanced mechanics of materials. 6th ed. ed. John Wiley \& Sons: New York; 2003.

[38] Neville A. Biology of Fibrous Composites: Development Beyond the Cell Membrane. New York, USA: Cambridge University Press; 1993. 
[39] Dalingwater, JE. Chelicerate Cuticle Structure. In: Nentwig, W, editor. Ecophysiology of Spiders: Springer-Verlag Berlin Heidelberg; 1987. p. 3-15.

[40] Schaber CF, Gorb SN, Barth FG. Force transformation in spider strain sensors: white light interferometry. J. R. Soc. Interface 2012;9:1254-64.

[41] Chizhik SA, Huang Z, Gorbunov VV, Myshkin NK, Tsukruk VV. Micromechanical Properties of Elastic Polymeric Materials As Probed by Scanning Force Microscopy. Langmuir 1998;14:2606-9.

[42] Kovalev A, Shulha H, Lemieux M, Myshkin N, Tsukruk VV. Nanomechanical probing of layered nanoscale polymer films with atomic force microscopy. J. Mater. Res. 2004;19:716-28.

[43] Hillerton, JE. Cuticle: Mechanical Properties. In: Bereiter-Hahn J, Matoltsy AG, Richards KS, editors. Biology of the Integument: Springer Berlin Heidelberg; 1984. p. 626-36.

[44] Barbakadze N, Enders S, Gorb S, Arzt E. Local mechanical properties of the head articulation cuticle in the beetle Pachnoda marginata (Coleoptera, Scarabaeidae). J. Exp. Biol. 2006;209:722-30.

[45] Barth F. Die Feinstruktur des Spinneninteguments. Z Zellforsch 1969;97:137-59.

[46] Vincent JFV, Wegst UGK. Design and mechanical properties of insect cuticle. Arthropod Structure \& Development 2004;33:187-99.

[47] Blickhan R. Stiffness of an arthropod leg joint. J. Biomechanics 1986;19:375-84.

[48] Thorson J, Biederman-Thorson M. Distributed Relaxation Processes in Sensory Adaptation. Science 1974;183:161-72.

[49] Mann DW, Chapman KM. Component mechanisms of sensitivity and adaptation in an insect mechanoreceptor. Brain Research 1975;97:331-6.

[50] Chapman K, Mosinger J, Duckrow RB. The role of distributed viscoelastic coupling in sensory adaptation in an insect mechanoreceptor. J. Comp. Physiol 1979;131:1-12.

[51] Bohnenberger J. Matched transfer characteristics of single units in a compound slit sense organ. J. Comp. Physiol 1981;142:391-402.

[52] Chyasnavichyus M, Young SL, Tsukruk VV. Probing of Polymer Surfaces in the Viscoelastic Regime. Langmuir 2014;30:10566-82. 

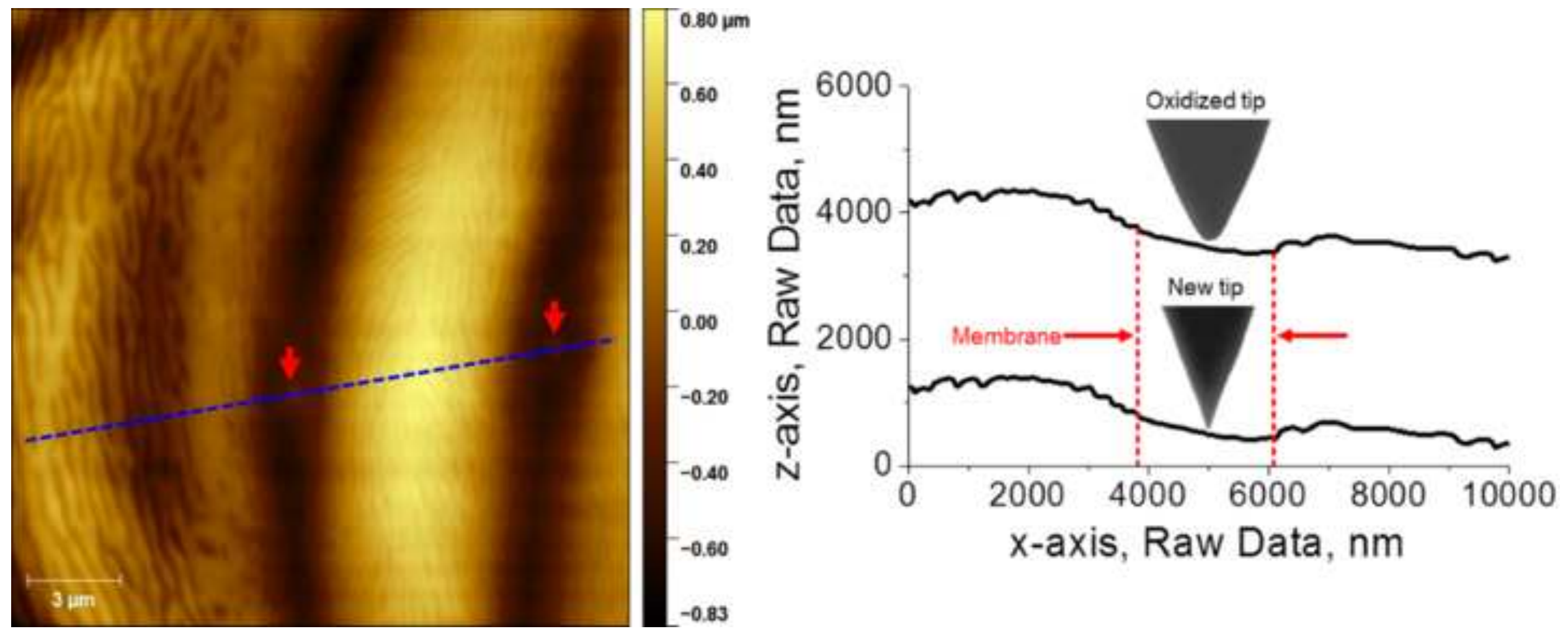